Comparative Population Studies

Selected Articles in German Vol. 40 (2015): 53-86

(Erstveröffentlichung: 03.06.2015)

\title{
Folgen der dauerhaft niedrigen Fertilität in Deutschland \\ Demografische Projektionen und Konsequenzen für unterschiedliche Politikfelder
}

\section{Martin Bujard}

Zusammenfassung: Deutschland ist länger als alle anderen Länder der Welt ein "Low-Fertility"-Land, seit vier Jahrzehnten liegt die Total Fertility Rate unterhalb von 1,5. Als "Pionier" in dieser Entwicklung lassen sich anhand einer Fallstudie Deutschlands die Folgen eines langfristigen Geburtenrückgangs analysieren. Dieser Rückgang ist in Deutschland zunehmend auch ein Megathema in Politik und Wissenschaft, da die Folgen sich auf mehrere Politikbereiche auswirken und teilweise bereits deutlich sichtbar sind. Allerdings werden diese Folgen völlig unterschiedlich eingeschätzt - zum einen in ihrer Vehemenz und danach, ob die Alterung oder die Schrumpfung das größere Problem ist. Nach beiden Prozessen differenziert, verbindet der Beitrag die demografische Analyse mit der politikwissenschaftlichen Bewertung der Folgen für die Politikfelder Rente, Gesundheit, Wirtschaft, Arbeitsmarkt, Kultur, Europäische Union, Internationale Beziehungen sowie den Parteienwettbewerb.

Die Folgen sind für alle diese Politikfelder gravierend, teilweise ambivalent, aber in der Summe negativ. Der Zeitpunkt der Folgen und die politischen Möglichkeiten damit umzugehen unterscheiden sich zwischen den Politikfeldern erheblich. Alterung ist ein größeres Problem als die Schrumpfung, da die vehementen Altersstruktureffekte für die Sozialversicherungen bis 2040 die meisten Deutschen betreffen und unabwendbar sind. Anders die Folgen der Schrumpfung, denn sie sind demografisch noch abwendbar und beträfen nicht alle Einwohner negativ. Bei einem Bevölkerungsrückgang muss man zwischen individueller und staatlicher Ebene differenzieren: Die Auswirkungen auf den Staat sind durch einen Rückgang an internationalem Einfluss und Macht, insbesondere in der Europäischen Union, negativ. Die Studie zeigt aber auch, dass die langfristigen Folgen des Geburtenrückgangs für den politischen Wettbewerb, die Kultur, den Arbeitsmarkt und die Wirtschaft nicht negativ sein müssen, wenn entsprechende Politikmaßnahmen erfolgen. Die Analyse identifiziert demografische und politische Stellschrauben für die Zukunft. Die Befunde dieser Fallstudie sind auch für andere Low-Fertility-Länder relevant, die einige Jahre später mit ähnlichen Prozessen konfrontiert sind.

Schlagwörter: Geburtenrückgang · Alterung · Sozialsysteme · Bevölkerungsrückgang · Europäische Union 


\section{$1 \quad$ Einleitung}

Im Jahr 2012 gab es weltweit bereits 29 „Low-Fertility“-Länder (World Bank 2014), wobei sich diese Definition an einer Total Fertility Rate (TFR) von unter 1,5 festmacht. Diese 29 Länder sind von dem Phänomen jedoch überwiegend erst seit ein bis zwei Jahrzehnten betroffen. Die Bundesrepublik Deutschland ist länger als alle anderen Länder, seit vier Jahrzehnten, ein Low-Fertility-Land. ${ }^{1}$ Nach Deutschland weisen Italien seit 1984, Spanien seit 1988 sowie Japan, Bulgarien und Rumänien seit 1993 am längsten ununterbrochen derart niedrige TFR auf. Für die Analyse der langfristigen Folgen des Geburtenrückgangs ist der Fall Deutschlands besonders interessant, weil bereits die zweite Generation von niedriger Fertilität betroffen ist, so dass sich die Folgen einer langfristig niedrigen TFR bereits stärker abzeichnen. Die folgende Fallstudie analysiert die Folgen des Geburtenrückgangs in Deutschland, wobei - trotz einiger spezifisch deutscher Rahmenbedingungen - die Erkenntnisse für viele andere Länder relevant sind, da diese zeitverzögert vor ähnlichen Herausforderungen stehen.

Der Geburtenrückgang und der demografische Wandel erfahren in Deutschland in Politik, Medien und Wissenschaft eine enorme Beachtung (vgl. Barlösius/Schiek 2007; Stock et al. 2012), inzwischen wird sogar von einem eigenen Politikfeld „Demografiepolitik" gesprochen (Hüther/Naegele 2013; Mayer 2012). In der Politik ist dieses Megathema inzwischen so angekommen, dass die Bundesregierung einen Demografiebericht (BMI 2012) erstellen ließ und eine Demografiestrategie (BMI 2013) entwickelt hat. Bundeskanzlerin Angela Merke/ stellt den demografischen Wandel auf die gleiche Stufe wie die Globalisierung (Merke/ 2012). Dass viele Politikfelder unmittelbar vom demografischen Wandel betroffen sind, zeigen nicht zuletzt die Mitwirkung fast aller Bundesministerien bei der Demografiestrategie der Bundesregierung und die Anwesenheit von acht Bundesministern und der Bundeskanzlerin bei den ersten beiden „Demografiegipfeln“ 2012 und 2013.

„Der demografische Wandel bedeutet neben den Fragen der Globalisierung wahrscheinlich die größte Veränderung unseres gesellschaftlichen Lebens, aber auch des persönlichen Lebens jedes Einzelnen in der ersten Hälfte des 21. Jahrhunderts." (Merkel 2012)

Angesichts dieser Bedeutung ist es erstaunlich, wie höchst unterschiedlich die Bewertungen der Folgen des Geburtenrückgangs in Deutschland sind. Während Birg (2003: 14) einen "demographischen Niedergang Deutschlands" sieht, der "Vorzeichen für den Abschied unseres Landes aus seiner tausendjährigen Geschichte" sein könnte, hält Hondrich (2007: 1) den Geburtenrückgang für einen „Glücksfall für unsere Gesellschaft". Die beiden Zitate weisen exemplarisch auf die enorme Bandbreite der Einschätzungen hin. Die Polarisierung zwischen "Untergangsszenarien“ und "Verharmlosung" ist charakteristisch für die Debatte über den Geburtenrückgang in den Arenen Politik, Medien und Wissenschaft in Deutschland.

1 Im Jahr 1975 beträgt die TFR 1,45 und ist seitdem mit Werten zwischen 1,24 und 1,45 bis heute unterhalb von 1,5 geblieben (Statistisches Bundesamt 2013a). 
Wie kommt das? Es gibt viele unterschiedliche Folgen für Gesellschaft, Wirtschaft und Politik, die bei einer Gesamtbewertung berücksichtigt werden müssen. $\mathrm{Zu}$ einzelnen Aspekten gibt es umfassende Analysen, jedoch wird meistens nicht zwischen beiden demografischen Konsequenzen des Geburtenrückgangs - Alterung $^{2}$ und Schrumpfung der Bevölkerungszahl - konsequent differenziert, was jedoch analytisch notwendig ist. Auch bei der Frage, ob die Folgen der Alterung oder die der Schrumpfung vehementer sind, unterscheiden sich die Einschätzungen in der Literatur fundamental.

Zudem müssen jeweils demografische Projektionen verwendet und deren Annahmen adäquat eingeschätzt werden. Häufig sind Analysen fragmentiert zwischen demografischen auf der einen Seite und sozial- bzw. politikwissenschaftlichen AnaIysen der Folgen anderseits. Das erschwert eine systematische Bestandsaufnahme der einzelnen Folgen des Geburtenrückgangs in Abhängigkeit von demografischen Parametern und damit eine wissenschaftlich fundierte Gesamtbewertung. Auch daher dominieren demografische Fehlinterpretationen, fragwürdige Annahmen oder partikuläre Betrachtungen die Einordnung des Phänomens Geburtenrückgang.

Dieser Artikel analysiert die Folgen des Geburtenrückgangs anhand einer Fallstudie zu Deutschland für verschiedene Politikfelder wie Rente, Gesundheit, Wirtschaft, Arbeitsmarkt, Internationale Beziehungen und EU sowie für die Gesellschaft und den parteipolitischen Wettbewerb. Dazu wird eine systematische Verbindung zwischen demografischen Prozessen einerseits (Abschnitt 3-4) und den politischen, gesellschaftlichen und ökonomischen Folgen andererseits (Abschnitt 5-6) hergestellt. Auf dieser Basis wird eine Synthese zu den Folgen der Geburtenrückgangs entwickelt, die dreierlei Fragen beantwortet: welche Folgen der Geburtenrückgang zu welchem Zeitpunkt auf die einzelnen Politikfelder hat, welche politischen und demografischen Stellschrauben in der Zukunft existieren und ob Alterung oder Schrumpfung problematischer ist. Abschließend wird die Übertragbarkeit der Befunde für andere Länder diskutiert.

\section{Forschungsstand und Analysestrategie}

\subsection{Forschungsstand}

Der Mitte der 1960er Jahre in Europa einsetzende Zweite Geburtenrückgang ist Teil des zweiten demografischen Übergangs (van de Kaa 1987). Er wird in der Demografie als eigenes Phänomen gegenüber dem ersten demografischen Übergang abgegrenzt, der von einem erheblichen Rückgang der Kindersterblichkeit begleitet wurde und in Deutschland von 1870 bis 1930 dauerte. Infolge des zweiten Geburtenrückgangs hat sich in Deutschland und einigen südeuropäischen und ostasiatischen Ländern die Total Fertility Rate (TFR) bei 1,2 bis 1,5 eingependelt, während

2 Der Begriff „Alterung“ wird hier für die Verschiebung in der Altersstruktur verwendet, wonach der Anteil älterer Menschen an der Bevölkerung deutlich zunimmt. 
andere Industrieländer in Nordeuropa, Nordamerika oder Frankreich eine TFR nahe des Bestandserhaltungsniveaus von 2,1 aufweisen (OECD 2014). Die TFR ist in vielen Ländern durch das steigende Alter der Frauen bei der Geburt beeinflusst und niedriger als die endgültige Kinderzahl der entsprechenden Kohorten (Bongaarts 2001). In der Bundesrepublik Deutschland liegt die TFR seit 1975 im engen Korridor von 1,24 bis 1,45, also seit mehr als einer Generation ein Drittel unterhalb der Ausgleichsrate (zu den Ursachen vgl. Bujard et al. 2012). Das ist weltweit die längste Phase eines solch niedrigen Geburtenniveaus.

Zur Analyse der Folgen dieses Geburtenrückgangs bedarf es einer analytischen Trennung zwischen Alterung und Schrumpfung (u.a. Swiaczny 2014). Beide Phänomene implizieren höchst unterschiedliche Folgen. Die Bewertung der Gesamtfolgen des Geburtenrückgangs in Deutschland variiert in der Literatur dahingehend, ob die Alterung die "schlimmere" Entwicklung sei (Sinn 2013) oder der Bevölkerungsrückgang (Birg 2003; Kaufmann 2005). Bei Bevölkerungsprojektionen unterscheidet sich der Einfluss der drei zentralen Parameter - Lebenserwartung, Migrationssaldo und Geburtenrate - im Hinblick auf Alterung und Schrumpfung fundamental.

Bevölkerungsprojektionen, die sowohl Projektionen für die BevölkerungsgröBe als auch die Alterung umfassen, gibt es u.a. von den Vereinten Nationen für den Zeitraum bis 2100 (UN 2012) und vom Statistischen Bundesamt (2009a/b) für Deutschland bis 2060. Dabei werden bisherige Trends der demografischen Parameter fortgeschrieben, wobei ein Spektrum unterschiedlicher Annahmen und ihrer Kombinationen berechnet wird. Diese Projektionen verstehen sich explizit nicht als Prognosen, trotzdem werden die mittleren Varianten faktisch oft als solche interpretiert. Allerdings sei auf zwei ernst zu nehmende Kritikpunkte an diesen Projektionen hingewiesen: Zum einen wird die Auswirkung des angenommenen durchschnittlichen Gebäralters auf das Fertilitätsniveau teilweise nicht berücksichtigt (Goldstein et al. 2011), was bei der Vorausberechnung des Statistischen Bundesamtes (2009a) angesichts eines ab 2020 nicht mehr steigenden Gebäralters in der mittleren Variante zu einer anschließenden Unterschätzung der TFR führen kann. Zum anderen kritisieren probabilistische Ansätze die deterministische Beschaffenheit klassischer Projektionen und dass die Unsicherheit durch verschiedene Varianten abgebildet und nicht anhand von Konfidenzintervallen quantifiziert wird (Werte für Deutschland siehe: Scherbov et al. 2008: 40-41). Allerdings können auch probabilistische Ansätze die Wahrscheinlichkeit von Systembrüchen und zukünftigen Entwicklungen der Annahmen nicht vorhersagen. Die Bevölkerungsvorausberechnungen des Statistischen Bundesamtes sind Grundlage der demografischen Analysen in Abschnitt 3 und 4, da sie in der Anwendung in Deutschland dominant sind.

Die Literatur zu den Folgen des Bevölkerungsrückgangs und der Alterung im Hinblick auf Sozialsysteme, Wirtschaft, Gesellschaft, EU, Internationale Beziehungen und den politischen Wettbewerb ist umfangreich. Da sich die bisherige Forschung oft auf einzelne Folgen bezieht, wird der Forschungsstand nicht gebündelt, sondern an entsprechender Stelle in Abschnitt 5 und 6 dargestellt. 


\subsection{Analysestrategie}

Um die Folgen des Geburtenrückgangs für ein bestimmtes Politikfeld zu analysieren, ist die Verbindung demografischer und politikwissenschaftlicher Perspektiven notwendig. Da der Geburtenrückgang sich mit Alterung und Schrumpfung in zwei unterschiedlichen demografischen Phänomenen manifestiert, werden diese Prozesse jeweils anhand verschiedener Zukunftsprojektionen diskutiert. Dabei werden die jeweiligen Dynamiken und Zeithorizonte sowie ihre Beeinflussbarkeit von den drei demografischen Hebeln Geburten, Migration und Lebenserwartung skizziert (siehe Abb. 1). Welchen Einfluss der Geburtenrückgang zu welchem Zeitpunkt und mit welcher Wahrscheinlichkeit bspw. auf die sozialen Sicherungssysteme hat, kann man nur verstehen, wenn man zuvor die demografischen Prozesse analysiert hat. Wohlgemerkt: Es reicht nicht aus, einen Projektionswert für ein bestimmtes Jahr zu betrachten, vielmehr ist ein Verständnis der Prozesse und Stellschrauben essentiell.

Abb. 1: Folgen eines Geburtenrückgangs mit jahrzehntelanger niedriger Fertilität

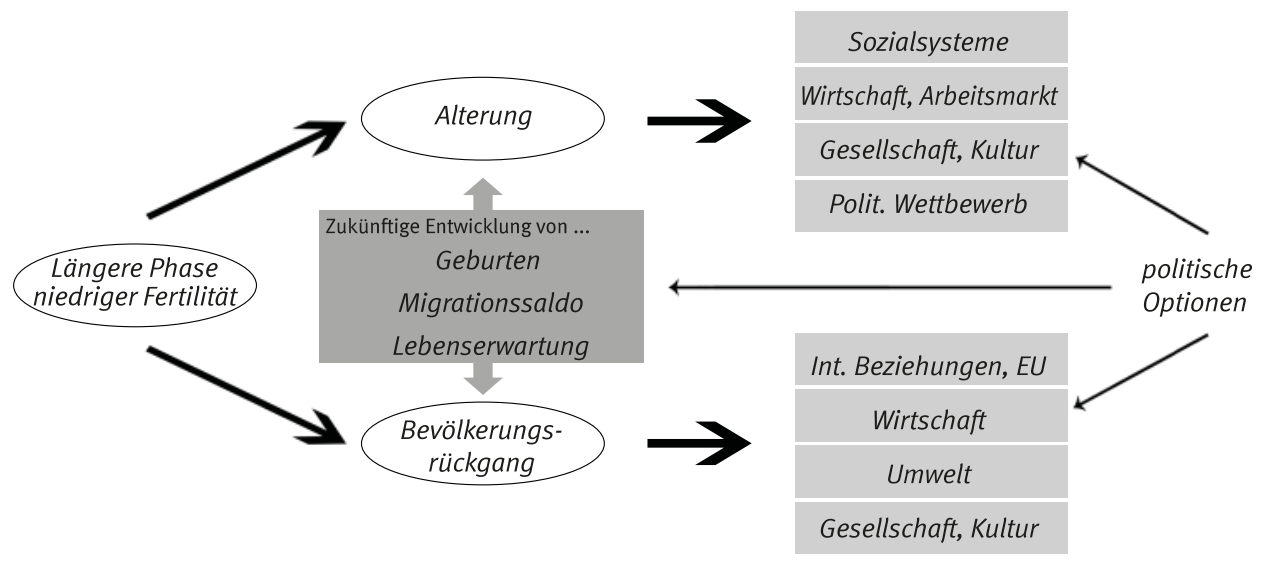

Quelle: Eigene Darstellung

Im nächsten Schritt werden die Folgen des Geburtenrückgangs für einzelne Politikfelder analysiert, wobei der Fokus auf einigen zentralen Folgen liegt. Die konsequente Trennung von Alterung und Schrumpfung wird beibehalten. Die Folgen der Alterung für Sozialsysteme, Wirtschaft, Gesellschaft und Parteiendemokratie werden anhand der unterschiedlichen Projektionen erörtert. Anschließend werden die Folgen des Bevölkerungsrückgangs in Hinblick auf Internationale Beziehungen, EU, Wirtschaft, Umwelt und Gesellschaft analysiert, gerade im Vergleich zu anderen Industrieländern. Diese Analyse ermöglicht auch eine Bewertung, welche Folgen als negativ, welche als positiv und welche nicht in dieser Dichotomie einordenbar sind.

Aufgrund des dynamischen Charakters demografischer Prozesse ist der Zeithorizont maßgeblich für die Beurteilung der Folgen. Diese sind dem Geburtenrückgang viele Jahrzehnte nachgelagert, wobei den Alterungsprozessen und dem Be- 
völkerungsrückgang eine unterschiedliche Dynamik und ein anderer Höhepunkt der Folgen zugrunde liegen. Hierbei ist ausschlaggebend, ob die Projektionen für den Zeitraum bis 2030, 2060 oder gar 2100 angefertigt sind. Da mit zunehmendem Zeitraum Projektionen unsicherer werden, andererseits aber die demografische Trägheit langfristige Berechnungen ermöglicht, wird der Frage nachgegangen, bis zu welchem Jahr und mit welchen Unsicherheiten diese sinnvoll zu interpretieren sind.

Anschließend wird die demografische Analyse mit der politikwissenschaftlichen zusammengebracht. Angesichts der Folgen des Geburtenrückgangs verbleiben zwei grundsätzlich verschiedene Arten politischer Optionen: die Beeinflussung der demografischen Parameter wie zukünftige Geburtenrate und Migration sowie die Anpassungsreaktionen in den einzelnen Politikfeldern. Dabei werden die jeweiligen Gestaltungsspielräume analysiert und eine Grundlage für demografiepolitische Politikberatung gelegt.

Diese Analyseschritte ermöglichen ein abschließendes Urteil zu den beiden vorne genannten Kontroversen: erstens zur Frage der Gesamtbewertung des Phänomens und zweitens, ob Alterung oder Bevölkerungsrückgang als gesamtgesellschaftlich negativer einzuschätzen sind.

\section{$3 \quad$ Alterung}

Die Alterung der Bevölkerung beruht nicht nur auf dem Geburtenrückgang, sondern wird durch die steigende Lebenserwartung verstärkt. Die Unterschiede der Altersstrukturen zwischen den Industrieländern sind weitestgehend auf den Geburtenrückgang zurückzuführen, da die Fertilitätsentwicklung zwischen den Ländern deutlich stärker variiert als der Trend der zunehmenden Lebenserwartung. Der Alterungsprozess lässt sich durch Lageparameter und Altenquotienten quantifizieren. So ist für die Auswirkungen der Alterung auf das Wahlsystem das Medienalter ausschlaggebend, während für die Sozialversicherungen der Altenquotient entscheidend ist.

\subsection{Medianalter}

Das Medianalter ist in Deutschland von 35 Jahren im Jahr 1950 auf 43 Jahre in 2009 gestiegen und wird zwischen 2045 und 2060 bei etwa 52 Jahren liegen. Das Medianalter der Wahlberechtigten ist für Veränderungen parteipolitischer Kräfteverhältnisse aufschlussreicher: Es war in der zweiten Hälfte des 20. Jahrhunderts recht konstant: es lag 1950 bei $44,4,1970$ bei $44,7,1990$ bei 45,0 und 2000 bei 46,4 Jahren. Im Jahr 2012 betrug es mit 50,1 erstmals mehr als 50 Jahre.

In Abbildung 2 ist das Medianalter der Wahlberechtigten bis 2060 dargestellt, das anhand der mittleren Variante (Untergrenze) der 12. koordinierten Bevölkerungsvorausberechnung (Statistisches Bundesamt 2009b) berechnet wurde. Demnach wird es 2030 auf 55,4 Jahre (vgl. Sánchez Gassen 2015: 248) und bis 2060 auf 58 Jahre steigen. Der Hauptanstieg erfolgt zwischen 2000 und 2030. Während bis in die 1990er Jahre die Hälfte der Wahlberechtigten unter 45 Jahre alt war, ist ab dem Jahr 2026 die Hälfte über 55 Jahre, also im Rentenalter oder kurz davor. 
Abb. 2: Medianalter der Gesamtbevölkerung und der Wahlberechtigten

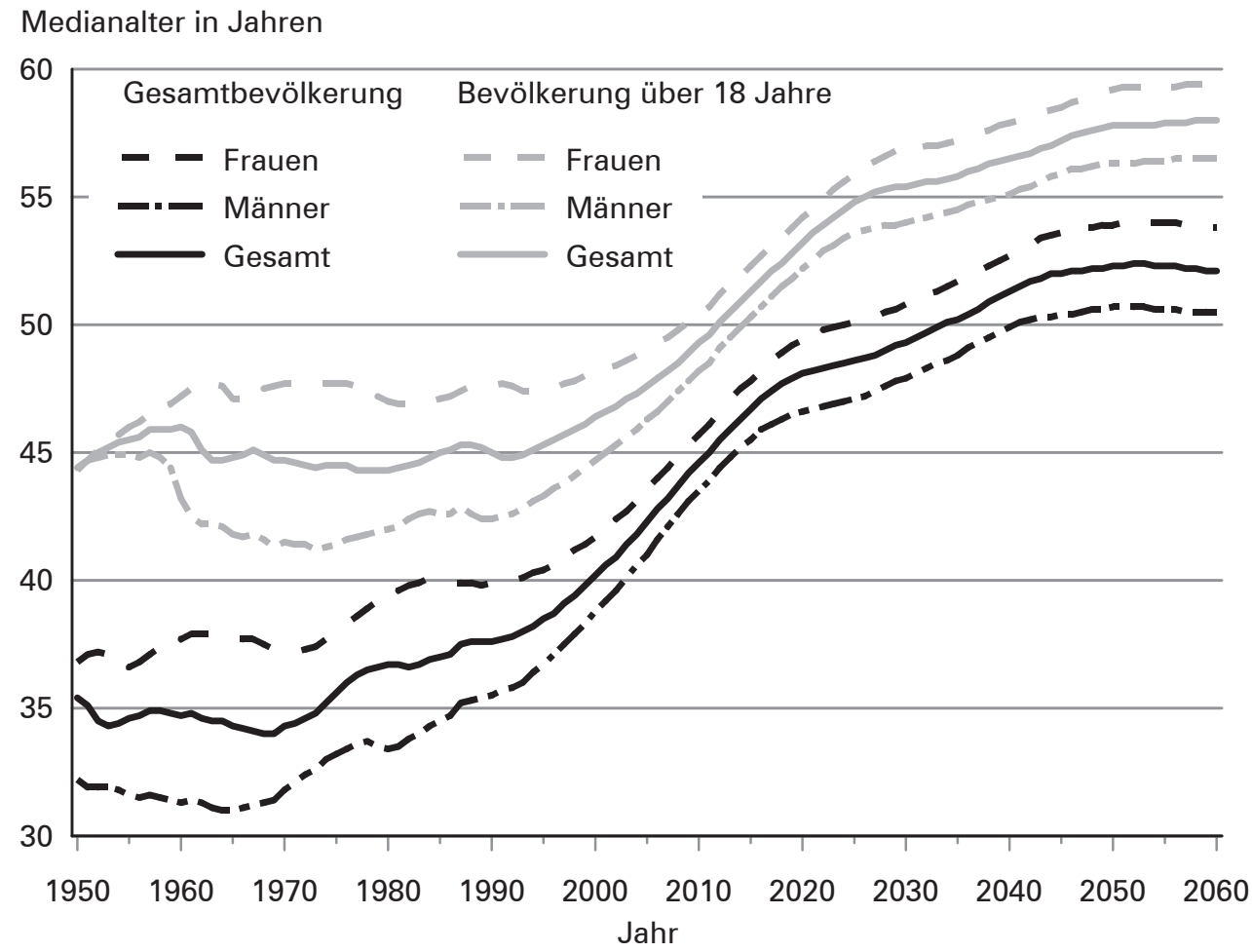

Anmerkungen: Ab 2012: Ergebnisse der 12. koordinierten Bevölkerungsvorausberechnung, Variante 1-W1 (Annahmen siehe Abb. 3). Die hier analysierte Bevölkerung ab 18 Jahre ist nicht identisch mit den Wahlberechtigten, da auch die nicht wahlberechtigte ausländische Bevölkerung inkludiert ist. Da die Altersstruktur bei den Deutschen etwas älter ist, ist das Medianalter der Wahlberechtigten minimal höher. Berechnungen für 2011 zeigen, dass dieser Effekt ein knappes Jahr (0,9 Jahre) ausmacht.

Quelle: Statistisches Bundesamt 2009b. Eigene Berechnung zusammen mit Harun Sulak.

\subsection{Altenquotient}

Der Altenquotient ist eine zentrale Maßzahl für die umlagefinanzierten Sozialversicherungen, er zeigt die Relation der Personen im Rentenalter zu denen im erwerbsfähigen Alter an. Der hier verwendete Altenquotient bezieht sich auf die Relation der ab 65-Jährigen zu den 20- bis 64-Jährigen. Der größte Anteil der epochalen Zunahme des Altenquotienten findet zwischen den Jahren 2010 und 2040 statt. In diesem Zeitraum erreichen die geburtenschwachen Jahrgänge den Arbeitsmarkt. Dieser Effekt wird durch die noch geburtenschwächere nächste Generation ebenso verstärkt wie durch das Erreichen des Rentenalters der Babyboom-Generation zwischen 2020 und 2030. Abbildung 3 zeigt das Projektionsspektrum des Statistischen Bundesamtes (2009a/b). Die Ergebnisse des neuen Zensus, die noch nicht in die- 
Abb. 3: Entwicklung des Altenquotienten auf Basis verschiedener Annahmen

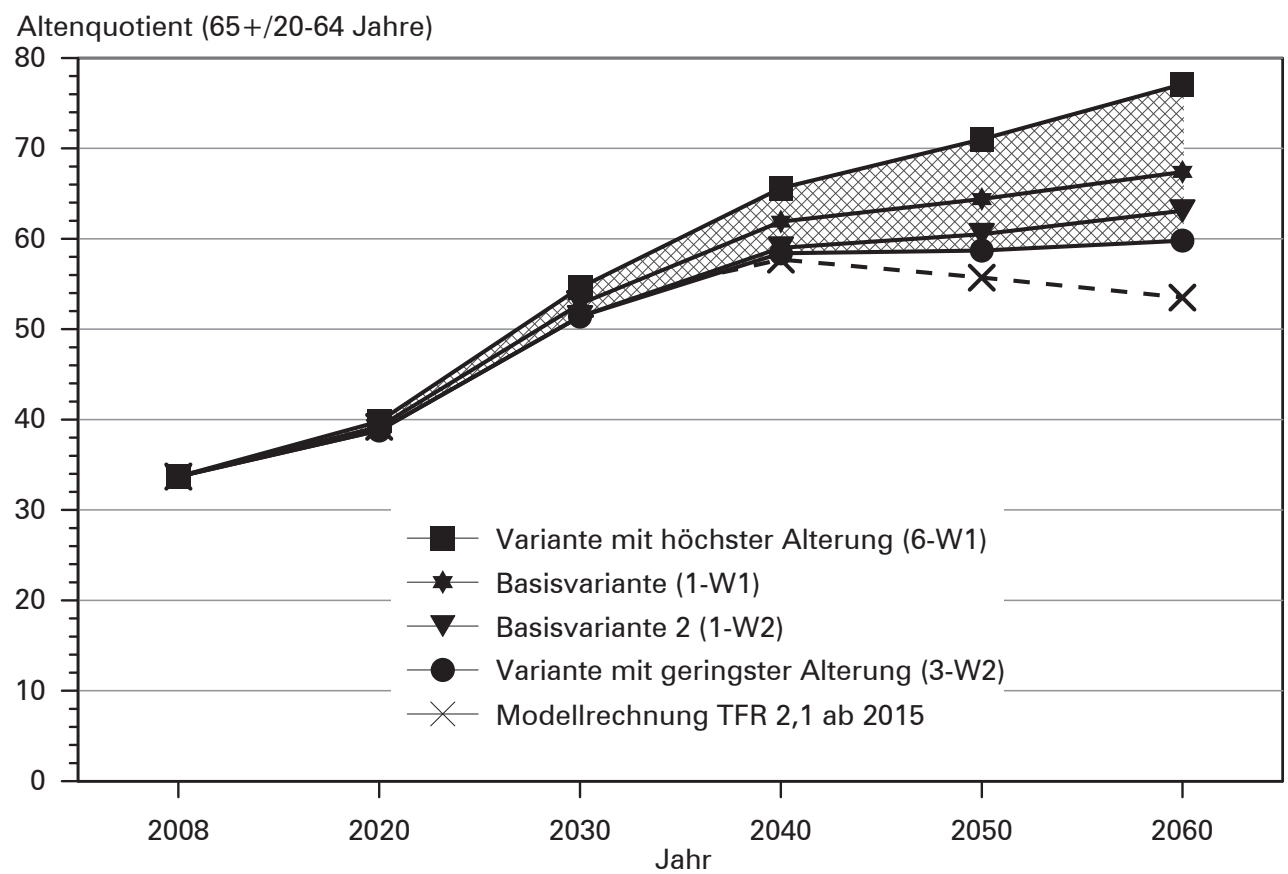

Anmerkungen: Annahmen der Basisvariante (1-W1): jährlicher Migrationssaldo langfristig 100.000, Lebenserwartung 2060 85,0 bzw. 89,2 Jahre; TFR 1,4. Basisvariante 2 (1-W2): Migrationssaldo langfristig 200.000, sonst gleich. Variante mit höchster Alterung (6-W1): TFR 1,2, Migrationssaldo langfristig 100.000, Lebenserwartung 87,7 bzw. 91,2 Jahre. Variante mit geringster Alterung (3-W2): TFR 1,6, Migrationssaldo langfristig 200.000, Lebenserwartung 85,0 bzw. 89,2 Jahre.

Quelle: Eigene Darstellung basierend auf Statistisches Bundesamt 2009b.

sen Bevölkerungsvorausberechnungen berücksichtigt sind, verzerren die Werte für den Altenquotienten nur unwesentlich. Das Spektrum der mittleren Basisvarianten weist Altenquotienten für 2060 von 63,1 und 67,4 auf, was ausgehend von 2008 einem Anstieg um 87 \% bzw. 100 \% entspricht. Die Verdopplung des Altenquotienten wird bereits 2040 mit Werten um 60 erreicht, danach flacht der Anstieg ab.

Im schattierten Bereich zeigt Abbildung 3 die Verläufe für die höchsten und niedrigsten Kombinationen von Annahmen zu Wanderung, Geburtenrate und Lebenserwartung innerhalb der zwölf berechneten und als realistisch eingestuften Varianten. In der Variante mit der höchsten Alterung kommt der Altenquotient 2040 auf 65,6 und steigt bis 2060 auf 77,1. Die Variante mit der geringsten Alterung, also der jüngsten Altersstruktur, verläuft bis 2040 mit 58,4 ähnlich und bleibt danach konstant (2060: 59,8).

Während sich der Anteil der ab 65-Jährigen bis 2040 verdoppelt, erhöht sich der Hochbetagtenanteil (ab 80-Jährige) aufgrund der steigenden Lebenserwartung noch stärker. Für Deutschland wird auf Basis der mittleren Vorausberechnungsva- 
riante (Statistisches Bundesamt 2009a) erwartet, dass sich der Hochbetagtenanteil von etwa $5 \%$ (4 Mio.) im Jahr 2008 auf $14 \%$ (9,05-9,23 Mio.) im Jahr 2060 fast verdreifachen wird.

\subsection{Einfluss der zukünftigen Migration und Geburtenrate auf die Alterung}

Wie wenig eine erhöhte Zuwanderung die Alterung bremsen kann, zeigt das viel zitierte Rechenbeispiel der Vereinten Nationen (UN 2000), wonach in Deutschland bis zum Jahr 2050 eine Zuwanderung in exorbitanter Höhe von 188 Millionen Menschen nötig wäre, um den Altenquotienten konstant zu halten. Zuwanderer und ihre etwas höhere Geburtenrate haben zwar Effekte auf Arbeitsmarkt und Altersstruktur (Sobotka 2008), werden später aber auch alt und ihre Geburtenrate nähert sich der deutschen häufig an (Birg 2003).

Im Folgenden wird der Hebel der Migration auf die Alterung quantifiziert: Wenn der jährliche Migrationssaldo bei 100.000 liegt, wird der Altenquotient 2060 der mittleren Projektion nach bei 67,4 liegen. Würde man unter sonst gleichen Bedingungen in jedem Jahr bis 2060 eine doppelt so hohe Zuwanderung annehmen, läge der Altenquotient bei 63,1 . Nach über 50 Jahren mit verdoppelter Migration hätte sich der Altenquotient also nur um 6,8\% reduziert.

Die Geburtenentwicklung der letzten fünf Jahrzehnte hat zwar die Alterung maßgeblich verursacht, allerdings könnte ein plötzlicher Anstieg der Geburtenrate an der Alterung kurz- und mittelfristig nur wenig ändern. Für die Altersstruktur ist die Zahl der Geburten und weniger die Geburtenrate entscheidend. Die Zahl der potenziellen Mütter im Jahr 2030 hängt von der Anzahl der in den letzten Jahren geborenen Mädchen ab. Da eine Generationenfolge etwa 30 Jahre umfasst und es weitere 20 Jahre dauert, bis das arbeitsfähige Alter erreicht ist, ist der Effekt der zukünftigen Geburtenentwicklung auf den Altenquotienten für die nächsten 50 Jahre relativ gering.

Würde die TFR statt bei rund 1,4 dauerhaft auf 1,6 ansteigen - was gegenwärtig etwa zusätzliche 96.000 Geburten pro Jahr wären - würde sich der Altenquotient bis 2060 nur von 67,4 auf 63,6 reduzieren. Ein halbes Jahrhundert mit deutlich höherer Geburtenrate würde den Altenquotienten also nur um $6 \%$ reduzieren. Würde die TFR dauerhaft auf 2,1 - die Ausgleichsrate - ansteigen, wäre der Altenquotient bis 2040 kaum anders. Zwischen 2040 und 2060 würde er dann jedoch nicht mehr weiter ansteigen, sondern leicht auf 53,5 zurückgehen (vgl. Abb. 3). Dies würde die Sozialsysteme ab 2040 entlasten. Verglichen zur Basisvariante (TFR 1,4) wäre der Altenquotient 2060 um 26,0 \% niedriger, so dass c.p. die Rentenbeiträge um ein gutes Viertel gesenkt (oder die Renten entsprechend erhöht) werden könnten.

\section{4 (Un-)Sicherheit von Projektionen zur Alterung}

Die Genauigkeit der hier skizzierten demografischen Projektionen ist aufgrund der demografischen Trägheit bis 2040 sehr hoch und bis 2060 relativ hoch. Selbst bei höchst unterschiedlichen Annahmen liegt der Altenquotient 2040 im engen Spekt- 
rum zwischen 58 und 66. Bis 2060 wird er in allen zwölf Projektionen ansteigen und zwischen 60 und 77 liegen. In absoluten Zahlen liegt dann das Spektrum der über 64-Jährigen zwischen 21,975 Mio. und 25,123 Mio. Bei weiterhin niedrigen Geburtenraten wird der Altenquotient jedoch nicht mehr so drastisch ansteigen wie zwischen 2010 und 2040, da die Nettoreproduktionsraten dann seit einem Menschenleben konstant niedrig sind. Allerdings könnte ein deutlicher Anstieg der TFR auf 2,1 ihn auf 53,5 bis 2060 senken und eine weitere Reduzierung in den Folgejahren bewirken.

Es kann also als sicher gelten, dass der durch den Zweiten Geburtenrückgang maßgeblich verursachte Alterungsprozess bis 2040 zu einer Verdoppelung des Altenquotienten, einer Verdreifachung des Anteils der Hochbetagten bis 2050 und einem Anstieg des Medianalters der Wahlberechtigten auf deutlich über 55 Jahre führt. Bis 2060 ist es wahrscheinlich, dass alle drei Indikatoren der Alterung grob auf diesem Niveau liegen, ob und wie stark sie weiter ansteigen, hängt von der zukünftigen Entwicklung von Geburten, Lebenserwartung und Migration ab.

\section{$4 \quad$ Bevölkerungsrückgang}

Der Bevölkerungsrückgang ist die zweite demografische Folge des Geburtenrückgangs. Geburtenraten unterhalb der Ausgleichsrate führen zu Nettoreproduktionsraten unterhalb von Eins. Da diese über mehrere Jahrzehnte in Deutschland existiert haben, ist ein niedriges Bevölkerungsmomentum entstanden (vgl. Blue/Espenshade 2011). In diesem Momentum, also in der Altersstruktur, ist ein Rückgang der Bevölkerung angelegt, der auch bei ansteigenden TFR noch viele Jahre weitergeht. Migration und steigende Lebenserwartung reduzieren den Bevölkerungsrückgang - anders als bei der Alterung. Auch die Dynamik im Zeitverlauf bei anhaltendem Fertilitätsniveau unterscheidet sich grundsätzlich: Im Unterschied zur Alterung setzt die Schrumpfung allmählich ein und verstärkt sich ab 2050 exponentiell.

\subsection{Projektionen der Bevölkerungsgröße bis 2060}

Abbildung 4 zeigt im grau schraffierten Bereich das Spektrum der zwölf vom Statistischen Bundesamt (2009a) als realistisch eingeschätzten Szenarien. Die darüber liegende Linie stammt aus einer Modellrechnung für eine TFR von 2,1, die als unrealistisch eingestuft wurde.

Die Bevölkerung geht in der Basisvariante (TFR 1,4; Lebenserwartung 85,0 und 89,2; Migrationssaldo 100.000) von 2010 bis 2030 um 4,2 Mio., bis 2040 um 7,7 Mio., bis 2050 um 12,1 Mio. und bis 2060 um 16,9 Mio. zurück. Deutschlands Bevölkerung hätte dann 2060 64,651 Mio. Einwohner - ein Rückgang um 20,7 \% innerhalb von 50 Jahren. In der Basisvariante 2, bei der der jährliche Migrationssaldo langfristig bei 200.000 liegt, wäre der Bevölkerungsrückgang erheblich geringer. Deutschland hätte dann 2060 70,120 Mio. Einwohner, was einem Rückgang von 11,4 Mio. bzw. $14 \%$ entspricht. Beide Szenarien sind in ähnlicher Weise denkbar bzw. realistisch, gleichzeitig aber kaum langfristig vorherzusehen. Betrachtet man sämtliche zwölf 
Abb. 4: Entwicklung der Bevölkerungsgröße Deutschlands auf Basis verschiedener Annahmen

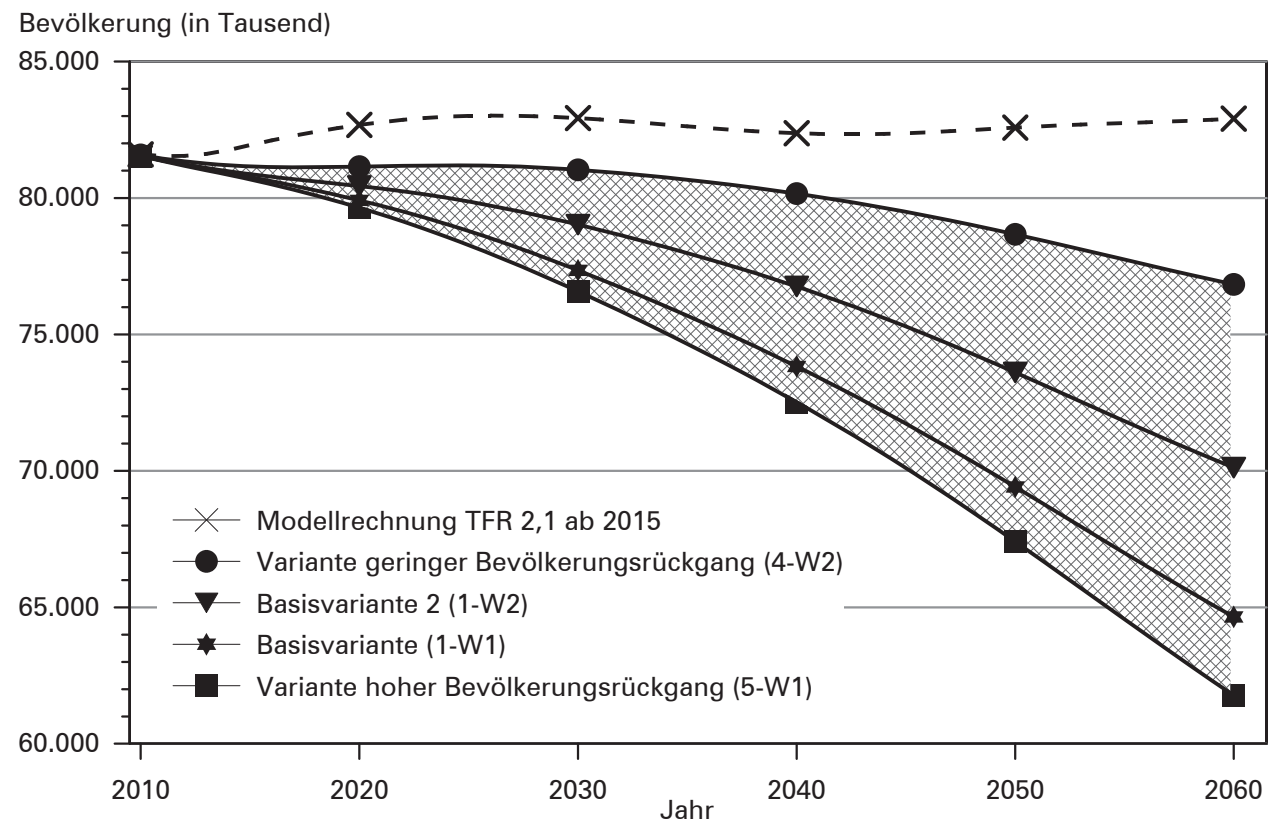

Anmerkung: Annahmen der Basisvarianten siehe Abb. 3.

Quelle: Eigene Darstellung basierend auf Statistisches Bundesamt 2009b.

Varianten, liegt die Bevölkerungszahl 2030 zwischen 76,6 und 81,0 Mio. und 2060 zwischen 61,8 und 76,8 Mio. Die Spannbreite der Projektionen ist erheblich: In einem Fall ist der Rückgang über einen langen Zeitraum von 50 Jahren minimal bei $5,9 \%$, beim anderen Extrem von 24,2 \% kann man von einem enormen Bevölkerungsrückgang sprechen.

Entscheidend für die konkreten Lebensbedingungen der Menschen ist allerdings die Bevölkerungsentwicklung auf regionaler Ebene. Hier gibt es gegenläufige Entwicklungen, bereits heute sind Wachstums- und Schrumpfungsregionen offenkundig. Aufgrund der fortschreitenden Binnenwanderung in Kombination mit der demografischen Verstärkung, die entsteht, wenn junge Menschen zum Beginn des Berufslebens abwandern, wird es in einigen Regionen Entvölkerungstendenzen geben (vgl. Kühntopf/Stedtfeld 2012). Da dieser Aspekt des demografischen Wandels nicht primär vom Geburtenrückgang induziert ist, wird er hier nicht weiter thematisiert. 


\subsection{Einfluss der zukünftigen Migration und Geburtenrate auf die Schrumpfung}

Die Bevölkerungsgröße bis 2060 wird von der heutigen Altersstruktur und der zukünftigen Entwicklung der drei Parameter Geburtenrate, Migration und Lebenserwartung beeinflusst. Ein weiterer Anstieg der Lebenserwartung ist unstrittig, der Einfluss ihrer unterschiedlichen Szenarien auf die Bevölkerungsgröße ist jedoch gering. Anders Migration und Geburtenrate:

Der Hebel der Migration auf die Schrumpfung lässt sich quantifizieren: Geht man von der Basisprojektion aus, würde bei einem jährlichen Migrationssaldo von 100.000 Menschen ab 2014 Deutschlands Bevölkerungsgröße 2060 bei 64,7 Mio. liegen. Würde man ab 2020 in jedem Jahr bis 2060 eine doppelt so hohe Zuwanderung annehmen, hätte Deutschland 70,1 Mio. Einwohner. 100.000 zusätzliche Migranten pro Jahr reduzieren den Bevölkerungsrückgang folglich um etwa ein Drittel. Mit entsprechend noch höherer Zuwanderung, grob geschätzt 400.000 pro Jahr, ließe sich die Schrumpfung bis 2060 komplett abwenden.

Die Annahme eines dauerhaften Zuwanderungssaldos von 100.000 oder gar 200.000 Menschen pro Jahr sollte kurz diskutiert werden: Eine derartige Zuwanderung von 2015 bis 2060 würde kumuliert einen Saldo von 4,6 bzw. 9,2 Mio. Menschen ausmachen. Da beim Saldo die Auswanderer von den Zuwanderern abgezogen werden müssen, wäre die Gesamtzuwanderung sogar deutlich höher. Da stellt sich die Frage, aus welchen Ländern diese Menschen kommen könnten. Historisch lag der Saldo zwischen 1950 und 2012 im Durchschnitt zwar bei 168.863 (eigene Berechnung nach: Statistisches Bundesamt 2013b), jedoch war die Entwicklung sehr volatil und von speziellen historischen Konstellationen wie dem Zuzug von Millionen (Spät-)Aussiedlern oder den EU-Erweiterungen geprägt. Da in den meisten klassischen Herkunftsländern die TFR inzwischen auch unter der Ausgleichsrate liegt, müsste eine hohe dauerhafte Zuwanderung zunehmend aus nicht-europäischen Ländern kommen. Dadurch würden sich die politischen und gesellschaftlichen Anforderungen an die Integration und die Anwerbung qualifizierter Zuwanderer vergleichsweise erschweren.

Nun zum Hebel der Geburtenrate: Wenn die TFR ab 2025 bei 1,6 statt 1,4 läge, kommt die Projektion 2060 auf 68,8 statt 64,7 Mio. Einwohner. Der TFR-Anstieg um 0,2 über 35 Jahre würde 4,1 Mio. Einwohner mehr ausmachen. Kombiniert man Migrations- und Geburtenhebel in der genannten Größenordnung und nimmt eine etwas optimistischere Lebenserwartung an, verstärken sich die Effekte, so dass die Schrumpfung bis 2060 bei dann 76,8 Mio. Einwohnern gering ist.

Der langfristige Hebel der TFR auf die Bevölkerungsgröße ist auch bei dem heutigen Altersaufbau immens. Angesichts manch apokalyptischer und fatalistischer Projektionen (u.a. Birg 2003) mag das Ergebnis überraschen: Wenn die TFR dauerhaft auf 2,1 steigen würde, würde die Bevölkerungszahl zwischen 2025 und 2027 sogar die 83-Mio.-Marke übersteigen und von 2028-2060 im engen Korridor zwischen 82 und 83 Mio. oszillieren. Bei dieser Berechnung wurden die Basisannahmen steigende Lebenserwartung (85,0 bzw. 89,2 Jahre) und Migrationssaldo von 100.000 
verwendet. Hier unterscheiden sich die Dynamik und das zukünftige Ausgleichspotenzial der demografischen Entwicklung grundlegend von der Alterung: Der Bevölkerungsrückgang ist auch heute komplett abwendbar, wenn die Geburtenrate auf das Ausgleichsniveau steigt. Je später sie das allerdings tut, desto stärker ist der zwischenzeitliche Bevölkerungsrückgang.

\subsection{Dynamik der Schrumpfung}

Um den exponentiellen Charakter des Bevölkerungsrückgangs darzustellen und die Effekte langfristig niedriger Geburtenraten einzuordnen, sind Modellrechnungen bis zum Jahr 2100 hilfreich. Diese sind allerdings nicht nur aufgrund des längeren Projektionshorizonts mit erheblichen Unsicherheiten verbunden, sondern auch, weil die schwer abschätzbare Höhe des Migrationssaldos und der TFR stärker als bei der Alterung die Projektionen beeinflusst. Hypothetisch lässt sich die Schrumpfungsdynamik verdeutlichen: Ohne Zuwanderung und bei dauerhaft niedrigen TFR würde sich jede Generation um rund $35 \%$ verkleinern, wobei sich diese Schrumpfungsspirale von Generation zu Generation beschleunigen würde.

Dieses wäre ein evolutionsbiologisch spannendes Phänomen, jedoch beruht es auf zwei diskussionswürdigen dauerhaften Annahmen: Die Annahme eines nicht positiven Zuwanderungssaldos entspricht weder der historischen Entwicklung der BRD bis in die Gegenwart, noch der relativen Attraktivität Deutschlands für Migranten. Gegen die Annahme langfristig andauernder niedriger Fertilität in der Zukunft könnten vier Gründe sprechen:

- der irgendwann nachlassende Verzerrungseffekt des Timings von Geburten auf die TFR (Bongaarts 2001),

- der intergenerationale Zusammenhang des generativen Verhaltens, wonach sich kinderlose Lebensstile weniger reproduzieren (Kolk et al. 2014),

- die langfristige Wirkung familienpolitischer Maßnahmen auf die Fertilität im internationalen Vergleich (Bujard 2011),

- die Einschätzung von Experten, deren Mittelwert für die TFR der Jahre 2030 und 2050 1,57 bzw. 1,58 beträgt (Basten et al. 2013: 68).

In den neuesten UN-Projektionen (UN 2012) gibt es eine Variante, bei der die durchschnittliche TFR konstant bis 2100 fortgeschrieben wird (Abb. 5). Bereits in der ersten Hälfte des 21. Jahrhunderts würden Frankreich und Großbritannien demnach Deutschland bei der Bevölkerungsgröße überholen. Da diese im Jahr 2050 bei allen drei Ländern ein ähnliches Niveau hat (69,0-72,9 Mio.), könnten leichte Veränderungen im Wanderungssaldo oder bei der Geburtenentwicklung die Reihenfolge verändern. Die Türkei würde 2020 mit Deutschland bei 81,2 Mio. Einwohnern gleichziehen und 2050 mit 103,4 Mio. etwa um die Hälfte größer sein. Im Jahr 2100 wäre dieser Modellrechnung nach die Türkei mit 122,1 Mio. weitaus größer als die anderen EU-Länder wie Frankreich $(77,8)$, Großbritannien $(75,1)$, Deutschland $(43,4)$, Italien (39) oder Spanien $(30,6)$. Mit Blick auf die historische Dimension ist festzustellen, dass dieser Bevölkerungsrückgang in seiner Art ein neues Phänomen in der 
Abb. 5: Bevölkerungsmodellrechnungen für die Jahre 2050 und 2100

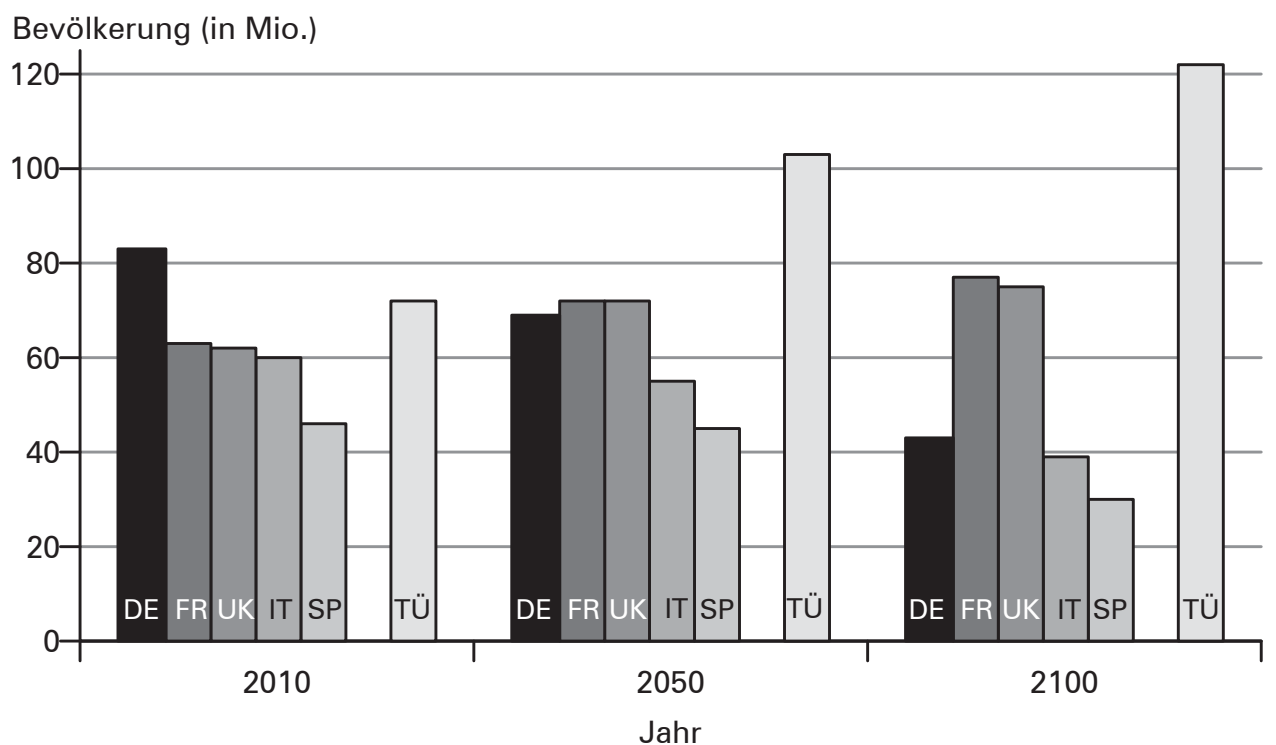

Quelle: Eigene Darstellung auf Basis von: UN (2012).

Weltgeschichte wäre. ${ }^{3}$ Anders die USA: Sie hätten aufgrund ihrer stationären TFR bei positivem Migrationssaldo dann mit 491,5 Mio. die 11-fache Bevölkerungsgröße Deutschlands.

Ob dieses Szenario eintrifft, weiß niemand. Entscheidend ist, ob sich die Geburtenrate in Deutschland wieder erhöht. Allerdings ist eine Schrumpfung bereits heute in der Altersstruktur angelegt: Obwohl Frankreich und die Türkei heute weniger Einwohner haben, ist die absolute Geburtenzahl dort deutlich größer als in Deutschland. Also steht heute schon fest, dass es in beiden Ländern in 30 Jahren mehr Frauen im gebärfähigen Alter gibt. In den letzten Jahren wurde der strukturell angelegte Bevölkerungsrückgang in Deutschland durch die Zuwanderung noch verschleiert: Beispielsweise lag das Geburtendefizit 2012 bei 196.038 (673.544 Lebendgeborene - 869.582 Gestorbene), durch den hohen Zuwanderungssaldo von 368.945, der durch die hohe Arbeitslosigkeit in vielen EU-Ländern bedingt ist, ist die Bevölkerung sogar um 172.907 angestiegen. In Jahren ohne großen Zuwanderungsüberschuss, wie 2006-2009, geht die Bevölkerung jedes Jahr um die Höhe des Geburtendefizits zurück. Deutschland schrumpft also derzeit ohne Einwanderung um rund 200.000 Menschen jedes Jahr. Dieser Trend setzt sich in den nächsten Jahren verstärkt fort. Der Basisvariante (1-W1) nach übersteigt das Geburtendefizit ab 2021 die 300.000er Schwelle, ab 2029 die 400.000er, ab 2041 die 500.000er und pendelt sich dann zwischen 500.000 und 600.000 ein. In jedem Jahr ab 2041 wird die Bevölkerung durch

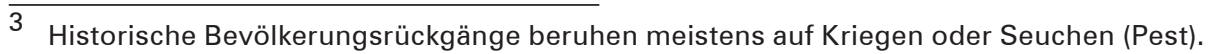


das Geburtendefizit um die Größe einer Stadt wie Düsseldorf, Leipzig, Bremen oder Nürnberg zurückgehen. Eine jährliche Zuwanderung in Höhe des durchschnittlichen Migrationssaldos der BRD seit 1950-2012 von 168.863 könnte nur ein knappes Drittel des Geburtendefizits kompensieren.

Zusammenfassend bleibt festzuhalten, dass Deutschland bei Verbleiben der Fertilität auf derzeitigem Niveau einen dramatischen Bevölkerungsrückgang erleben wird. Die deutsche Altersstruktur ist auf einen Rückgang angelegt, das Geburtendefizit verstärkt sich aufgrund einer sich beschleunigenden Dynamik von Jahr zu Jahr. Der Bevölkerungsrückgang jedoch kann durch eine Erhöhung der Geburtenraten ebenso abgebremst werden, wie durch eine kontinuierliche hohe Zuwanderung.

\section{$5 \quad$ Die Folgen der Alterung}

Die Auswirkungen des Alterungsprozesses sind für mehrere Politikbereiche von immenser Tragweite. Insbesondere die Sozialversicherungen sind davon massiv betroffen (u.a. Castles 2004; Schmid et al. 2000). In diesem Abschnitt werden die wichtigsten Folgen für die Sozialsysteme, die wirtschaftliche Entwicklung und den Arbeitsmarkt skizziert, aber auch mögliche gesellschaftliche und kulturelle Veränderungen sowie Implikationen für den politischen Wettbewerb.

\subsection{Die Folgen der Alterung für die Sozialsysteme}

Die erwartete Verdoppelung des Altenquotienten von etwa 2010 bis 2040 hat unmittelbare Konsequenzen für die Sozialversicherungen. Die Probleme sind bei umlagefinanzierten Rentensystemen größer, ebenso bei ehrgeizigen Sozialsystemen mit hohem Dekommodifizierungsgrad und in Ländern mit geringer Geburtenrate. Daher sind die Rentenprobleme in Deutschland besonders ausgeprägt. Im Folgenden werden Rentenproblematik und potenzielle Lösungsansätze anhand der Rentenformel ausführlicher dargestellt. Die Rentenformel setzt sich vereinfacht aus diesen fünf Variablen zusammen:

$$
b=A Q * N * \frac{a(R)}{a(B)} * \frac{1}{S}
$$

Der Beitragssatz (b) entspricht dem Altenquotienten (AQ) multipliziert mit dem Rentenniveau (N) multipliziert mit dem Quotienten aus Rentner-Koeffizienten ${ }^{4}(a(R))$ und Erwerbstätigen-Koeffizienten $(a(B))$ multipliziert mit dem Kehrwert des Steueranteils (S). Ein Gedankenspiel anhand der Rentenformel: Wenn sich der Altenquotient verdoppelt und alles andere konstant bleibt, verdoppelt sich automatisch der Beitragssatz - derartig hohe Lohnnebenkosten hätten dramatische Folgen für den

4 Der Anteil der Rentner an der ab 60-(65-)jährigen Bevölkerung im Verhältnis zu den Erwerbstätigen in dieser Altersklasse. 
Arbeitsmarkt. Möchte man den Beitragssatz konstant halten, würde sich c.p. das Rentenniveau halbieren - verbreitete Altersarmut wäre die Folge. Eine Anpassung über das Renteneintrittsalter würde ein tatsächliches Eintrittsalter von 71 Jahren erfordern, was einem formellen von etwa 75 Jahren entspricht (vgl. Rürup/Schmidt 2003) - dies ist illusorisch. Auch der Steueranteil allein könnte diese Dimension nicht annähernd ausgleichen.

Als Reaktion auf die vorausberechnete Verdoppelung des Altenquotienten zwischen den Jahren 2000 und 2035 wurde in Deutschland an allen vier verbleibenden Stellschrauben der Rentenformel gedreht. Betrachtet man die fast jährlichen, inkrementalistischen Reformen im Rentensystem, so kann man diese Sparmaßnahmen jeweils leicht einer dieser vier Stellschrauben zuordnen:

1) Die erste Stellschraube ist eine Erhöhung des Beitragssatzes, wie es seit 1953 27-mal geschah (Schmidt 2005). Dies ist die offensichtlichste Stellschraube. Für die Jüngeren kommen mit staatlichen Zuschüssen unterstütze Investitionen in kapitalgedeckte Rentenversicherungen zu den Beitragssätzen hinzu. Die Internationalisierung der Wirtschaft setzt der Finanzierung zu Lasten der Lohnnebenkosten jedoch faktische Grenzen (Scharpf 2001).

2) Eine geringere Anrechnung von Ausbildungsjahren, die Anbindung an die Nettolohnentwicklung, der Blüm'sche demografische Faktor 1997, der ähnliche Schröder'sche Nachhaltigkeitsfaktor 2004, eine Reduzierung der Anrechnung für Schul- und Hochschulausbildung und die Entrichtung des vollen Pflegebeitrags für Rentner mindern das Rentenniveau mit der Stellschraube zwei. Die Niveausicherungsklausel nach § 154 Abs. 3 SGB VI sichert lediglich die Brutto-Standardrente eines Rentners mit 45 Jahren Durchschnittsverdienst auf dem Niveau von $43 \%$ im Jahr 2030 (derzeit $52 \%$ ).

3) Die dritte Stellschraube, die Veränderung des Rentner-Koeffizienten, wird durch die schrittweise Anhebung des formellen Ruhestandsalters von 65 auf 67 Jahre ab dem Jahr 2011 bis 2029 justiert. Auch wurde die Frühverrentung in Deutschland erschwert wie bspw. durch den § 77 des SGB VI, wonach der Zugangsfaktor sich um 0,3 \% pro vorzeitigen Monat Ruhestand reduziert. Des Weiteren entlastet ein Anstieg der Arbeitsmarktpartizipation in der erwerbsfähigen Bevölkerung die Sozialsysteme, u.a. durch eine Erhöhung der Frauenerwerbstätigkeit und den Abbau der Arbeitslosigkeit. Walter et al. (2013: 42) zeigen Szenarien, wonach eine Kombination dieser Faktoren den Rückgang der Erwerbspersonenzahl bis 2030 reduzieren kann.

4) Steuergelder fließen beispielsweise durch die Ökosteuer in das Rentensystem. Umgekehrt liegt durch die Finanzierung von Beamtenpensionen bereits eine demografisch bedingte wachsende Last auf dem Staatshaushalt. Ein Transfer des Steuersystems in das Rentensystem ist z.B. die Herausnahme versicherungsfremder Leistungen.

Durch die langfristigen Reformen, durch die Erhöhung des Rentenzugangsalters auf 67 Jahre und des Nachhaltigkeitsfaktors ist das deutsche Rentensystem bereits zu einem beträchtlichen Teil an das Ansteigen des Altenquotienten in den nächsten Jahrzehnten angepasst worden. Dabei hat die deutsche Alterssicherungspolitik einen einnahmeorientierten Charakter angenommen zu Lasten der Orientierung an einem gewünschten Niveau. Jedoch sind die sozialen Belastungen für Beitragszahler und Rentner, aber auch die makroökonomischen Belastungen für Staat und Wirt- 
schaft bis zum Jahr 2040 exorbitant hoch. Das Argument, wonach die durch den Geburtenrückgang induzierte Rentenproblematik durch Produktivitätsfortschritte ausgeglichen werden könnte (u.a. Hondrich 2007), ist kaum haltbar. Dazu ist erstens die Dimension der Verschiebung des Altenquotienten zu hoch und zweitens ist es naiv anzunehmen, dass Unternehmen und Arbeitnehmer sämtliche Produktivitätsgewinne über Jahrzehnte nur in die Rentenkassen fließen lassen.

Die Folgen der Alterung für die gesetzliche Kranken- und Pflegeversicherung (GKV, GPV) ähneln denen für die Alterssicherung insofern, da auch hier weniger Beitragszahler auf mehr Leistungsempfänger kommen. Die Größenordnung ist für die Lohnnebenkosten zwar annähernd gleich hoch, allerdings sind die Folgen des Geburtenrückgangs aus zwei Gründen hier geringer: Die Rentner sind an der Finanzierung der GKV selbst beteiligt, und die fernere Lebenserwartung nach dem Renteneintritt ist nicht mit Krankheitsjahren gleichzusetzen. Grundsätzlich sind die Gesundheitsausgaben schwieriger zu prognostizieren, da Angebot und Nachfrage von Gesundheitsdienstleistungen nicht allein auf demografischen Annahmen beruhen - vor allem der medizinische Fortschritt fungiert als Kostentreiber. Birg (2003: 185) errechnet, dass sich der Beitragssatz der Krankenkassen bis 2050 von $13 \%$ auf 21-24 \% erhöhen und der Pflegesatz auf rund $6 \%$ vervierfachen wird. Allerdings sind in Relation zum BIP, anders als beim Beitragssatz, die Leistungsausgaben der GKV in Deutschland seit drei Jahrzehnten konstant geblieben (Rürup/Ranscht 2009: 112). Anders der Anstieg der Pflegekosten: Die drastische Zunahme der Hochbetagtenquote führt zu einer Zunahme bestimmter pflegebedürftiger Krankheitsbilder wie Demenz, so dass trotz medizinischer Verbesserungen eine hohe Steigerung wahrscheinlich ist (vgl. Dob/hammer/Scholz 2010). Hier muss man allerdings zwischen professioneller und informeller Pflege differenzieren (Michee/ 2013). Diesen Überlegungen folgt, dass selbst bei einem deutlichen Anstieg der öffentlichen Gesundheitsausgaben eine Zunahme an Eigenverantwortung und eine partielle Rationalisierung der Leistungen auf die Menschen zukommen wird. Diese Entwicklung birgt große sozialpolitische Konflikte.

Aufgrund des Geburtenrückgangs muss die Gesellschaft in den nächsten drei Jahrzehnten also einen wachsenden Teil der Produktivität und des Wohlstands in die Sozialsysteme umschichten und trotzdem werden Rentenniveau und GKVLeistungen relativ abnehmen. Daran könnte auch ein zeitnaher Anstieg der Geburtenrate nichts ändern. Nach 2040 wird der Altenquotient weniger schnell steigen, wodurch der Anpassungsdruck auf die Sozialsysteme dann nachlassen wird.

\subsection{Die Folgen der Alterung für Wirtschaft und Arbeitsmarkt}

Steigende Sozialabgaben bewirken indirekt eine Belastung für Wirtschaft und Arbeitsmarkt - insbesondere für Wohlfahrtsstaaten, die ehrgeizig sind und sich in starkem Maße über den Faktor Arbeit finanzieren (Schmidt 2005). Insofern könnten die oben skizzierten sozialstaatlichen Folgen negative Effekte auf den Arbeitsmarkt haben, was jedoch politisch steuerbar ist, wenn diese nicht überwiegend auf die Lohnnebenkosten abgewälzt werden. Gelegentlich wird argumentiert, dass sich durch das schrumpfende Arbeitsangebot von Erwerbstätigen die Arbeitslosigkeit 
reduziert. Dies folgt jedoch einem statischen Verständnis von Angebot und Nachfrage, da diese Effekte höchstens kurzfristig auftreten können (Friedman 1968).

Inwieweit sich die Produktivität einer älteren Belegschaft verändert, wird kontrovers diskutiert. Die These, Alterung gehe mit einer geringeren Innovationsfreude einher, lässt sich nicht belegen. Teilweise werden Forschungen von Guilford zitiert (Sinn 2003: 67), nach denen die größte geistige Leistungskraft mit 30-40 Jahren erreicht wird. Diese These ist jedoch zu undifferenziert, da bei älteren Arbeitnehmern Wissens- und Erfahrungskomponenten zunehmen (Akademiegruppe Altern 2009: 41). Zudem steigt die Fitness der 60-Jährigen stetig an. Ein zentraler Punkt ist politisch steuerbar: Investitionen in Humankapital können auch bei älteren Arbeitskräften in Form zunehmender Weiterbildungsmaßnahmen eine innovationssteigernde Wirkung haben.

Die Auswirkungen auf das Wirtschaftswachstum lassen sich anhand der CobbDouglas-Produktionsfunktion nachvollziehen, wonach sich das Produkt aus dem technischen Fortschritt, dem Arbeitseinsatz und dem Kapitaleinsatz zusammensetzt. Der Arbeitseinsatz in Form der Anzahl der Menschen im erwerbsfähigen Alter geht in den nächsten drei Jahrzehnten durch den bisherigen Geburtenrückgang absolut und relativ zurück. Dieser Effekt könnte durch umso größeren technischen Fortschritt oder Kapitaleinsatz kompensiert werden. Nach verschiedenen Modellrechnungen könnte die wirtschaftliche Wachstumsrate Europas dauerhaft zurückgehen: laut OECD (1998) von 2,3 \% auf 0,5 \% für 2025-2050. Durch eine Kapital-Intensivierung (Lee/Mason 2007) oder durch zunehmende Bildungsinvestitionen (Lutz et al. 2008) lassen sich negative Wachstumseffekte der Alterung jedoch kompensieren. Striessnig und Lutz (2013) zeigen anhand von Simulationsmodellen mit um Bildung gewichteten Unterstützungskoeffizienten, dass auch Fertilitätsraten unterhalb der Ausgleichsrate optimal sein könnten. Die Fokussierung der demografischen Folgen auf das Wachstum des Sozialprodukts unterliegt einer Beschränkung, da es nicht mit dem Wachstum von Lebensqualität gleichzusetzen ist (Easterlin 1974).

Unterhalb dieser Gesamteffekte induziert die Alterung diverse sektorale Nachfrageverschiebungen (Kaufmann 2005). So werden Pflegedienstleistungen, Tourismus, Altenheime und Finanzdienstleistungen in der Zukunft Wachstumsindustrien sein. Auch steigt infolge der Alterung das Erbschaftsvolumen, wodurch sich dem Staat eine große Einnahmequelle eröffnet und das pro Kopf gerechnete Vermögen ansteigt.

\subsection{Die Folgen der Alterung für Gesellschaft und Kultur}

Hinsichtlich der gestiegenen Lebenserwartung und der Kompression der Morbidität auf wenige Jahre ist Alterung prinzipiell ein für viele Menschen vorteilhaftes Phänomen im Sinne "gewonnener Jahre" (Imhof 1981). Durch den Geburtenrückgang entsteht zusätzlich jedoch eine Verschiebung der Kohortengrößen zwischen Jung und Alt, deren Folgen für die Gesellschaft ambivalent und spekulativ sind. In alternden Gesellschaften könnte zwar die Risikoaversion höher sein (Kaufmann 2005), andererseits hat dies eine enorm stabilitätssichernde Wirkung im Vergleich zu extrem jungen Gesellschaften (Huntington 1996). Die These einer quantitativen Abnahme 
von Verwandtschaft und des familiären Netzwerks (u.a. Schirrmacher 2004) ist angesichts demografischer Vorausberechnungen zu hinterfragen (Dude/ 2014).

Die Begriffe "Alter", "Überalterung" und "alternde Gesellschaft" sind überwiegend negativ und teilweise mit Klischees besetzt (Akademiegruppe Altern 2009). Schirrmacher (2004) skizziert Szenarien, wonach ein "Krieg der Generationen“ und "rassistische Altersstereotypen“ die Zukunft prägen. Kruse (2013) dagegen verweist auf Potenziale wie differenzierte Wissenssysteme, reflektierte Erfahrungen, Resilienz, Generativität und Gerotranszendenz. Höhn et al. (2006) zeigen, dass die Menschen in den OECD-Ländern nicht altenfeindlich eingestellt sind und die Erfahrung älterer Menschen geschätzt wird. Außerdem ist zu beachten, dass 70-Jährige in der Zukunft viel gesünder, mobiler und selbstständiger als 70-Jährige in früheren Zeiten sind. Insofern bietet der dritte Lebensabschnitt von Pensionären bzw. Rentenbeziehern enorme Chancen, sowohl individuell als auch zivilgesellschaftlich, bspw. durch ehrenamtliches Engagement dieser Altersgruppe. Zudem existieren vielfältige Beziehungen und Transfers in den intergenerationalen Beziehungen, bei denen die ältere Generation ein großes Potenzial an Teilhabe und Unterstützung aufweist, wobei die jeweilige Beziehungsqualität ein maßgeblicher Faktor ist (ausführlich siehe: Ette et al. 2010). Die gesellschaftlichen Folgen der Alterung sind spekulativ und hängen davon ab, wie Politik und Gesellschaft damit umgehen. Dabei fordert die Akademiegruppe Altern (2009) auf der individuellen Ebene ein Umdenken bezüglich tradierter Lebenslaufvorstellungen, auf der Ebene der Unternehmen kontinuierliche Investitionen in Qualifikation und auf der gesellschaftlichen Ebene die Überwindung eines negativen Altersbildes.

\subsection{Die Folgen der Alterung für den politischen Wettbewerb}

Wie oben gezeigt wurde, steigt das Medianalter der Wahlberechtigten bis 2040 so weit an, dass Rentner und kurz vor der Rente stehende Personen eine Mehrheit im Wahlsystem haben. Damit treffen Menschen, die nicht mehr erwerbstätig sind, Entscheidungen, deren Folgen von Erwerbstätigen zu tragen sind. Die Frage ist, wie sich diese Interessen im Parteiensystem und der politischen Willensbildung auswirken werden - ob sie gar zu einer "Gerontokratie" führen.

Langfristige Bindungen von Wählern an Parteien und Milieus und systemerhaltene Adaption der Parteien sprechen dafür, dass Renteninteressen innerhalb des Parteiensystems vertreten werden. Das Alter ist auch schwer als Konfliktlinie vorzustellen, da innerhalb eines Lebenslaufes meistens beide Seiten vorkommen (Goerres 2007). Allerdings könnten gerade die Volksparteien SPD und CDU/CSU zu Parteien mutieren, die die Rentnerinteressen in besonderem Maße vertreten, zumal die Rentner ihr treuestes Wahlklientel sind. Ein Beispiel ist der Koalitionsvertrag der dritten großen Koalition, der eine Mütterrente für Geburten vor 1992 und die Rente mit 63 nach 45 Beitragsjahren vorsieht (CDU, CSU, SPD 2013). Für die Volksparteien birgt eine zu starke Orientierung an Rentnerinteressen die Gefahr, dass sich eine der kleineren Parteien als Sprachrohr der unter 50-Jährigen profiliert. Bereits heute sind Unterschiede auf Einstellungsebene zur Rentenpolitik und Wählerverhalten zwischen Alt und Jung evident (Bergmann et al. 2012). 
In der Zukunft sind verschiedene Szenarien vorstellbar: Die Konfliktlinie Alt/Jung bzw. Sozialstaatsempfänger/-finanzierer könnte sich im Parteiensystem und in der Verbändestruktur abbilden, so dass der Verteilungskampf in verschärfter Form über den Parteienwettbewerb und in polarisierender Rhetorik über die Medien ausgetragen wird. Die Präferenz für Sozialausgaben wie Rente oder Bildung ist in hohem Maße altersabhängig (Busemeyer et al. 2009). Möglich wäre jedoch auch, dass dieser Konflikt durch die etablierten Parteien und Interessengruppen aufgegriffen und kanalisiert wird, so dass ältere Konfliktlinien dominant bleiben. Da alte Menschen ein großes Interesse am Wohlergehen der jüngeren Generation zeigen, besonders wenn sie selbst Kinder und Enkel haben, ist auch ein relativ konfliktfreier Interessenausgleich zwischen den Generationen denkbar (vgl. Bergmann et al. 2012). Insofern wäre eine Konfliktlinie zwischen Kinderlosen und Familien eher wahrscheinlich, da bei ersteren der generationenverbindende Verwandtschaftseffekt so nicht gegeben ist. Generationenverbindend wäre auch eine als geburtenfördernd kommunizierte und am Kindeswohl orientierte nachhaltige Familienpolitik, von der die junge Generation direkt und die ältere indirekt über den nachlassenden demografischen Problemdruck profitiert.

\section{$6 \quad$ Die Folgen des Bevölkerungsrückgangs}

\subsection{Die Folgen des Bevölkerungsrückgangs für die Internationalen Beziehungen und die EU}

Für demografische Effekte auf die außen- und verteidigungspolitische Stärke eines Landes gibt es mehrere historische Beispiele. Einen machtpolitischen Rückgang hat Davis (1945: 603) den Europäern bereits 1945 prophezeit. Der Einfluss der Verschiebungen der demografischen Gewichte zwischen Frankreich, Deutschland und Russland auf die militärische Stärke im 19. und frühen 20. Jahrhundert wurde oft thematisiert und auch im Zusammenhang mit dem Ausbruch des Ersten Weltkrieges diskutiert (Morgenthau 1948: 123). Auch in der merkantilistischen Epoche oder in der Antike beim Untergang Roms spielte der wirtschafts- und machtpolitische Aspekt von Bevölkerungsgröße eine wichtige Rolle.

Zweifellos wird der Bevölkerungsrückgang in den besonders betroffenen Staaten Europas und Ostasiens zu einem relativen Verlust an außenpolitischer Bedeutung führen. Formulierungen wie "demografischer Niedergang Deutschlands und Europas" (Birg 2003: 14) sind auf Basis demografischer Analysen jedoch nicht haltbar. Doch die "Verschiebung internationaler Gewichte" (Wöhlcke et al. 2004: 11) hat politikwissenschaftlich interessante Implikationen für die internationalen $\mathrm{Be}$ ziehungen. Ein Nexus von Bevölkerungsrückgang und Machtverlust lässt sich aus (neo)realistischer Perspektive (Morgenthau 1948) aus der Schwächung von militärischer Stärke herleiten und aus interdependenztheoretischer Sicht (Keohane/Nye 1977) aus dem sinkenden Gewicht in internationalen Organisationen wie UN, NATO, aber auch EU. Die Bevölkerungsgröße wirkt sich unmittelbar da aus, wo eine Legitimation nach dem demokratischen Prinzip hergeleitet wird - beispielsweise der 
doppelten Mehrheit bei EU-Ratsentscheidungen. Zudem reduzieren sich bei einem Bevölkerungsrückgang die Wirtschaftskraft und damit auch die militärischen Möglichkeiten - insbesondere in Relation zu den Staaten, deren Bevölkerung und Wirtschaftskraft zunehmen. Ob sich die EU neben den USA und China als Weltmacht etablieren kann, wird nicht an der demografischen Entwicklung scheitern. Die EU-28 würde auch bei konstanter TFR 2100 noch über 300 Mio. Einwohner haben. Vielmehr ist die Entwicklung der politischen Kriterien ausschlaggebend, also inwieweit eine außen- und verteidigungspolitische Integration in der EU zu "politischer Handlungsfähigkeit, politischem Konsens und Ordnungserfahrung" (Weidenfeld 1995) führt.

Innerhalb der EU wird die demografische Entwicklung Deutschlands gegenwärtige Dominanz dauerhaft reduzieren. Deutschland ist nicht nur das ökonomisch stärkste Mitglied, sondern hat auch mit Abstand von fast 20 Mio. auf Frankreich und Großbritannien die größte Bevölkerung. Im Jahr 2030 wird der Abstand auf unter 10 Mio. geschmolzen sein und in den 2040ern sind alle drei Staaten auf dem gleichen Niveau, möglicherweise überholen beide Deutschland in 30 Jahren. Bei den Effekten auf Deutschlands Stellung in der EU gilt es zwischen dem demokratischen Prinzip und der ökonomischen Stärke zu differenzieren: Im EU-Ministerrat wird sich die Machtbalance in den nächsten Jahrzehnten verschieben, da die Mehrheitsentscheidungen eine doppelte Mehrheit von $55 \%$ der EU-Staaten und $65 \%$ der EUBevölkerung erfordern. Dies führt zu neuen Gestaltungsmehrheiten und Sperrminoritäten (Münch/Wilkoszewski 2006), wobei Deutschlands Einfluss entsprechend der oben genannten Projektionen zurückgehen wird. Analoges gilt für Deutschlands Repräsentation im Europäischen Parlament (EP). Zweitens reduziert der Geburtenrückgang die gesamte Wirtschaftskraft des Landes stärker als die pro Kopf berechnete. Bei aller Ungewissheit über die langfristige ökonomische Entwicklung ist - demografisch bedingt - ein erheblicher Rückgang der ökonomischen Vormachtstellung Deutschlands plausibel. Vor diesem Hintergrund hat die gegenwärtige EU-Binnenmigration nach Deutschland enorm weitreichende außenpolitische Implikationen.

\subsection{Die Folgen des Bevölkerungsrückgangs für die Wirtschaft}

Der Nexus zwischen Bevölkerungsgröße und Ökonomie ist Gegenstand mehrerer ökonomischer Theorien. Nach Adam Smith (1776) verstärkt Bevölkerungswachstum die Potenziale an Arbeitsteilung und Skaleneffekten. Der Umkehrschluss bei Bevölkerungsrückgang ist bei großen Binnenmärkten wie der EU und globalisierten Gütermärkten jedoch von untergeordneter Bedeutung. Keynes sieht in dem damaligen leichten Bevölkerungsrückgang eine Mitursache für die Nachfrageschwäche in der Großen Depression. Mehrere neoklassische Ansätze externalisieren die Bevölkerungsentwicklung (u.a. Friedman 1968). Die Wachstumstheorie von Solow (1956) analysiert dagegen den wechselseitigen Zusammenhang von Bevölkerungswachstum, Einkommen und Investitionen. Dabei sinkt der Pro-Kopf-Kapitalstock bei steigendem Bevölkerungswachstum, und umgekehrt kann ein Bevölkerungsrückgang demnach c. p. zu einer Erhöhung der Pro-Kopf-Investitionen und des Sozialprodukts pro Kopf (BIPPC) beitragen. Der Mangel an Arbeitskräften ist aus neoklassischer Perspektive durch erhöhten Kapitaleinsatz substituierbar. Demnach kann das ge- 
samte BIP bei einem Bevölkerungsrückgang dann wachsen, wenn technischer Fortschritt und Investitionen ihn überkompensieren.

Für technischen Fortschritt sind jedoch nicht nur Kapitalinvestitionen, sondern vor allem gute Ausbildung möglichst vieler Arbeitskräfte von Bedeutung. Dieses thematisiert die Humankapitaltheorie (Schultz 1971). Da sich Humankapital aus dem Produkt von Arbeitskräften und ihrer durchschnittlichen Qualifikation zusammensetzt, kann ein Bevölkerungsrückgang mit Bildungsinvestitionen theoretisch kompensiert werden (Lutz et al. 2008). Dazu müssten die Bildungsinvestitionen in Ländern mit einer TFR um 1,4 aber weitaus höher sein als in Ländern mit höherer Fertilität. Da dies in Deutschland trotz einer Nettoreproduktionsrate von 0,59 bis 0,69 seit 1975 nicht der Fall war, errechnet Kaufmann (2005) eine Investitionslücke in das Humankapital von 2,5 Billionen Euro.

Grundsätzlich ist jedoch zu hinterfragen, die wirtschaftlichen Folgen am Wachstum des Sozialproduktes eines Landes festzumachen: Das BIPPC ist für die Prosperität und den zukünftigen Wohlstand viel aussagekräftiger als das gesamte BIP, denn bei einem Bevölkerungsrückgang ist es vorstellbar, dass das BIP fällt, während das BIPPC ansteigt.

Wenn man statt des Wachstums den Vermögensstand einer Volkswirtschaft betrachtet, kommt man zu einem positiveren Ergebnis, denn das Vermögen pro Kopf steigt c.p., wenn der Nenner kleiner wird. Aber auch die öffentlichen Schulden erhöhen sich bei einem Bevölkerungsrückgang, wenn man sie pro Kopf betrachtet. Beides zusammen legt den Schluss nahe, dass dann eine stärkere Besteuerung des Vermögens sinnvoll wäre. Ein anderer wichtiger Aspekt betrifft die Konzentration von Vermögen und die Verteilungsgerechtigkeit, die zukünftig stärker durch eine Besteuerung von Erbschaftsvermögen hergestellt werden muss. Die Vererbung an eigene Kinder wird für einen zunehmenden Personenkreis nicht möglich sein, da der Anteil kinderloser Frauen und Männer auf über $20 \%$ angestiegen ist (bezogen auf die 1960er Kohorten, vgl. Statistisches Bundesamt 2013a). Bei dieser Gruppe könnte das Erbschaftssteuervolumen relativ größer sein, wenn man die Besteuerung von Erbschaften an direkte Nachkommen durch Freibeträge begünstigt. Betrachtet man Wohlstand aus der Perspektive von Sen (1995), der die Teilhabechancen betont, sind sogar positive Effekte möglich: Aufgrund der kleineren Kohorten und dem Bedarf an gut ausgebildeten Arbeitnehmern gibt es ökonomische Anreize, die bildungsfernen Schichten stärker als heute auszubilden und zur Teilhabe zu „befähigen".

Der Bevölkerungsrückgang wird zu einem gigantischen Strukturwandel führen, der besonders in ländlichen Regionen dramatische Ausmaße annehmen wird. Aufgrund von Binnenwanderung, v. a. der jungen Bevölkerung in urbane Regionen mit Arbeitsplätzen, wird der Bevölkerungsrückgang in manchen urbanen Regionen kompensiert und in deindustrialisierten und ländlichen Regionen noch verstärkt. Dieser Effekt ist bereits heute in Ostdeutschland sichtbar. Aber auch einige Städte werden deutlich schrumpfen. Diese regionalen Disparitäten beinhalten große Herausforderungen für die öffentliche Daseinsfürsorge, v.a. für Dienstleistungsangebote und öffentliche Infrastruktur wie Schulen, Kindergärten, Krankenhäuser, Pflegeeinrichtungen oder Feuerwehr (vgl. BMI 2012; Steinführer et al. 2014). Die Folgen 
für Miet- und Immobilienmärkte der ländlichen Schrumpfungsregionen sind erheblich, für Mieter positiv und für Immobilienbesitzer negativ.

\subsection{Die Folgen des Bevölkerungsrückgangs für die Umwelt}

Die Auswirkungen des Bevölkerungsrückgangs für Energie und Umwelt sind dagegen positiv, da der Ressourcenverbrauch und die Umweltverschmutzung bei einer geringeren Bevölkerungszahl ceteris paribus abnehmen. Auf nationaler Ebene könnte ein Bevölkerungsrückgang zur Renaturalisierung in einigen Regionen genutzt werden. Weitaus bedeutender ist hier jedoch die globale Perspektive, da der Ressourcenverbrauch in Industrieländern wie Deutschland überproportional hoch ist (siehe auch: Meadows et al. 1972). Der pro Kopf bezogene Ressourcenverbrauch in den Industrieländern hat seit der Meadows-Publikation bezüglich der meisten Ressourcenarten zugenommen. Der ökologische Fußabdruck ist pro Person von 1961 bis 2007 in Europa um 30 \% und in Deutschland um 42 \% angestiegen (Ewing et al. 2010: 63). Ein Maßstab für Nachhaltigkeit ist, dass sich die genutzten Umweltressourcen langfristig regenerieren lassen. In einer globalen Perspektive ist dies nicht der Fall, was sich v.a. durch den Klimawandel und den Verlust an Biodiversität sowie durch eine zunehmende Ressourcenknappheit an Energie, Wasser oder Fischbestand auswirkt. Hier kann ein Bevölkerungsrückgang auch eine Chance sein, den Ressourcenverbrauch und den Klimawandel zu reduzieren.

Aus globaler Perspektive sind die Einsparpotenziale Deutschlands allerdings zu relativieren: Denn erstens macht die Bevölkerung Deutschlands nur 1,1\% der Weltbevölkerung aus und dieser Anteil ist rückläufig (UN 2012) und zweitens nimmt der Ressourcenverbrauch in den Entwicklungs- und Schwellenländern, in denen der größte Teil der Weltbevölkerung lebt, enorm zu. Folgende Zahlen zum Ressourcenverbrauch gemessen in globalen Hektar pro Person (gha) im Jahr 2007 verdeutlichen die Relationen: Der ökologische Fußabdruck der Produktion liegt in Deutschland bei 4,72 gha, der des Konsums bei 5,08 gha, während die Biokapazität 1,92 beträgt. Dieses pro Kopf berechnete Defizit multipliziert sich mit der Bevölkerungszahl. In Deutschland wird also das 2,65-fache an Ressourcen im Vergleich zur Biokapazität verbraucht. Global werden "nur" das 1,52-fache der Ressourcen verbraucht (Ewing et al. 2010: 31). Demnach ist das deutsche Defizit mit $165 \%$ mehr als drei Mal so hoch wie das globale von $52 \%$. Beim Bevölkerungsrückgang steigt nach diesem Modell die Biokapazität pro Kopf an.

Neben diesem Effekt ist die Symbolwirkung nicht zu unterschätzen, wenn sich in einem Industrieland der Ressourcenverbrauch bzw. die Bevölkerungsgröße reduziert. Dazu kommt, dass sich die Befunde auch auf andere Länder übertragen lassen und ein Bevölkerungsrückgang in mehreren Industrieländern einen erheblichen Entlastungsfaktor für die Umwelt bedeuten könnte. Abgesehen von dieser demografischen Argumentation gibt es andere politische und technologische Hebel, um die Umweltbelastung zu reduzieren. 


\subsection{Die Folgen des Bevölkerungsrückgangs für die Gesellschaft und Kultur}

Die kulturellen Folgen sind schwer abschätzbar. Die meisten gesellschaftlichen Institutionen wie Kirchen, Gewerkschaften, Parteien und Verbände werden einen Mitgliederschwund erleiden. Der Begriff „Entvölkerung“ oder gar die Analogie mit dem Dreißigjährigen Krieg (Schmid et al. 2000: 184) sind per se nicht angemessen angesichts eines Bevölkerungsrückgangs bis 2060 nach den mittleren Projektionen um 14 bis $21 \%$. Allerdings gibt es Regionen, in denen Abwanderung und Geburtendefizit zusammenkommen, was sich für die Bleibenden als dramatische Entvölkerung darstellt. Dies ist insbesondere in einigen ostdeutschen Regionen der Fall, wobei nicht nur die Ost-West-Migration, sondern auch Wanderungen zwischen ostdeutschen Regionen ursächlich sind (Sander 2014). Welche gesellschaftlichen Auswirkungen das haben wird, hängt auch von der kompensierenden Regionalpolitik ab. Neben diesen Verliererregionen wird es prosperierende Städte und Regionen geben, deren Bevölkerung in den nächsten Jahrzehnten nicht zurückgehen wird.

Träger von Kultur und Sprache eines Landes ist die Bevölkerung. Wenn diese schrumpft, schrumpft auch die Anzahl der Menschen, die die Sprache verstehen und sprechen sowie die kulturellen Traditionen leben und weitergeben. Die die kollektive Identität formenden Kommunikations-, Erfahrungs- und Erinnerungsgemeinschaften (Kielmannsegg 1995) werden durch den Bevölkerungsrückgang kleiner. Wie sehr eine zurückgehende kulturelle Bedeutung im internationalen Vergleich oder eine geringere Trägerschaft kultureller und historischer Erfahrungen als negativ empfunden wird, wird sehr unterschiedlich bewertet. Der Versuch, angesichts der konstanten Zuwanderung Menschen mit Migrationshintergrund über Generationen hinweg dauerhaft aus diesen Kommunikationsgemeinschaften auszuklammern (und sei es durch Bevölkerungsberechnungen, vgl. Birg 2003), ist abwegig - nicht nur, da er fehlenden Integrationswillen und wirkungslose Integrationspolitik für die Zukunft unterstellt, sondern auch angesichts von gemischten Familiengründungen und Auswirkungen des Staatsbürgerschaftsrechts.

\section{$7 \quad$ Fazit, Synthese und Diskussion}

Die Fallstudie Deutschland zeigt, dass die Folgen der jahrzehntelang niedrigen Geburtenrate in erheblichem Maße von politischen Weichenstellungen, aber auch von der zukünftigen Migrationsrate abhängen. Die extremen Einschätzungen einiger Autoren sind nicht zutreffend. Die deutsche Geburtenentwicklung ist weder ein "Glücksfall“, noch wird sie einen "Niedergang Deutschlands" hervorrufen. Der Geburtenrückgang führt zu den beiden demografischen Prozessen Alterung und Bevölkerungsrückgang, die sich massiv auf die gesellschaftliche, politische und ökonomische Zukunftsentwicklung auswirken. Intensität, Zeitpunkt und Bewertung der Folgen sind je nach Politikfeld verschieden. In der Gesamtbewertung sind die Folgen überwiegend negativ, teilweise ambivalent und sie manifestieren sich langfristig mit großer Vehemenz. Dies widerspricht den zunehmend auftretenden Be- 
wertungen, die eine neutrale Gesamtbilanz der Folgen des Geburtenrückgangs mit Herausforderungen und Chancen vornehmen. Andererseits sind die Folgen für den politischen Wettbewerb, die Kultur, den Arbeitsmarkt und die Wirtschaft nicht zwingend negativ, da sie von politischen Entscheidungen und Bewertungsperspektiven abhängen.

Hinter dieser Gesamtbewertung sind fundamentale Differenzierungen bezüglich demografischer Projektionen sowie politischer, ökonomischer und kultureller Folgen notwendig:

(1) Manche demografische Folgen sind sicher, andere beruhen auf Szenarien, von denen wir nicht wissen, ob sie eintreffen. Nahezu sicher ist die Alterung, insbesondere dass sich der Altenquotient von 2000 bis 2040 mehr als verdoppelt. Der Altenquotient 20-65, der 2008 bei 33,7 lag, wird 2040 etwa zwischen 57 und 66 liegen. Sicher ist auch der Anstieg des Medianalters der Wahlberechtigten von heute 50,1 auf etwa 55 Jahre bis 2030 mit steigender Tendenz in den Folgejahren. Anders der Bevölkerungsrückgang: Es ist heutzutage sogar noch offen, ob und in welchem Ausmaß wir ihn erleben werden. Bei einem zeitnahen Anstieg der TFR auf 2,1 und einem geringen Migrationssaldo von 100.000 würde die Bevölkerung bis 2060 um 82-83 Mio. liegen. Dieses Szenario ist nicht das wahrscheinlichste, aber auch nicht völlig unrealistisch. In realistischeren Szenarien mit einer TFR zwischen 1,2 und 1,6 wird die Bevölkerung 2040 zwischen 74 und 78 Mio. und 2060 zwischen 61 und 77 Mio. liegen. Sollte jedoch die TFR die nächsten 86 Jahre auf dem heutigen niedrigen Niveau verbleiben und der Migrationssaldo nicht steigen, würde die deutsche Bevölkerung erheblich auf 43,4 Mio. schrumpfen - jedoch sind derart langfristige Modellrechnungen höchst spekulativ.

(2) Einige politische Entwicklungen lassen sich so gestalten, dass sie sich nicht per se in die Dichotomie "positiv-negativ" einordnen lassen; andere wiederum sind bei allen Gestaltungsspielräumen negativ. Die deutlich negativste Entwicklung betrifft die Folgen für die Sozialsysteme. Auch wenn sich die Halbierung der Renten (die c.p. der Verdopplung des Altenquotienten entspräche) durch die Kombination von höherem realen wie formellen Renteneintrittsalter, Steuerzuschüssen, Beitragssatzanstieg und höherer Frauenerwerbstätigkeit vermeiden lässt und Horrorszenarien abwendbar sind: Der Geburtenrückgang reduziert den zukünftigen Wohlstand erheblich. Denn der Anstieg des realen Renteneintrittsalters und der Frauenerwerbstätigkeit würde ohne die Alterung zu höheren Renten und niedrigeren Lohnnebenkosten führen. Ab 2030 werden für mehrere Jahrzehnte die Renten niedriger und die Krankenkassenleistungen begrenzter sein, was zu zunehmender Altersarmut und Exklusion von einigen Gesundheitsleistungen führt. Zudem wird bei den arbeitenden Generationen ein erheblicher Teil der Produktivitätszuwächse für die sozialen Sicherungssysteme verwendet werden.

Die Folgen für den politischen Wettbewerb ergeben sich durch den Anstieg des Medianalters der Wahlberechtigten: Das Wahlgewicht der Rentner nimmt rapide zu. Inwieweit dies zu Reformblockaden oder Veränderungen im Parteiensystem führt, bleibt abzuwarten. Dafür sprechen die gemeinsamen pekuniären Interessen von Rentnern in Verteilungsfragen - dagegen die Adaptionsfähigkeit von Parteien und der intergenerationalen Solidarität der Wähler. Innerhalb der EU wird das relative Gewicht Deutschlands infolge des Geburtenrückgangs abnehmen. In den 2040er Jahren werden Frankreich und Großbritannien eine ähnliche Bevölkerungsgröße wie Deutschland haben. Dies wirkt sich direkt über die doppelte Mehrheit im Rat und das Abgeordne- 
tenkontingent im EP aus, zudem indirekt über Verhandlungspositionen und Wirtschaftskraft.

(3) Die ökonomischen Folgen des Geburtenrückgangs sind unterschiedlich: Die Effekte für das Potenzialwachstum und das BIP sind tendenziell negativ aufgrund des höheren Abhängigkeitsquotienten (Alterung) und des Rückgangs des Humankapitals (Bevölkerungsrückgang). Allerdings lässt sich der Rückgang der arbeitenden Bevölkerung durch eine höhere Arbeitsmarktbeteiligung, einen höheren Kapitalstock und v.a. höhere Bildung kompensieren. Negative Gesamteffekte auf das BIPPC sind jedoch nicht plausibel. Je stärker der Bevölkerungsrückgang sein wird, desto mehr steigen die Staatsverschuldung pro Kopf, aber auch das Vermögen pro Kopf. Die sektoralen Nachfrageverschiebungen werden erheblich sein. Regional wirkt sich der Bevölkerungsrückgang aufgrund von Binnenwanderungen sehr unterschiedlich aus. Es wird entleerte Regionen mit einbrechenden Immobilienpreisen und regionalpolitischen Herausforderungen ebenso geben wie prosperierende Städte.

(4) Die kulturellen Folgen der Alterung werden vielfältig sein. Negative Altersstereotype sind weitgehend unrealistisch, da Rentner gesünder und vitaler sein werden als in früheren Generationen. Das zivilgesellschaftliche Engagement und die öffentliche Wahrnehmung der Rentnergeneration werden zunehmen. Das Ausmaß eines kulturellen Verlustes im Falle eines erheblichen Geburtenrückgangs - eines möglichen, jedoch nicht sicheren Szenarios - sind normativ schwer einzuschätzen. Träger von Sprache und Erinnerungsgemeinschaften würden sich reduzieren. Dies wird sich im kulturellen Alltag kaum auswirken, jedoch würde die internationale "Geltung" erheblich zurückgehen. Da gleichzeitig der Anteil von Menschen mit Migrationshintergrund zunehmen wird, wird auch die Kultur vielfältiger. Bewertungen dazu sind oftmals subjektiv, auf jeden Fall kommt der Integrationspolitik und dem Spracherwerb eine zunehmend wichtige Rolle zu. Kommunikations-, Erlebnis- und Erinnerungsgemeinschaften können sich sowohl auf vergangene Jahrhunderte als auch auf kürzere Zeiträume, die Menschen mit und ohne Migrationshintergrund gemeinsam haben, beziehen.

Tabelle 1 fasst für die analysierten Politikfelder jeweils die demografische Wahrscheinlichkeit bestimmter Folgen, den Zeithorizont, die politischen Optionen und eine Bewertung der Folgen zusammen.

Die Frage, ob Alterung oder Bevölkerungsrückgang die schlimmere Folge des Geburtenrückgangs für die Menschen ist, lässt sich hier klar beantworten: Alterung. Erstens weil die Verdopplung des Altenquotienten bis 2040 sicher ist, während ein Bevölkerungsrückgang um mehr als $20 \%$ keineswegs sicher ist. Zweitens da sich die Alterung massiv auf die Sozialsysteme und damit auf den Wohlstand fast aller Deutschen auswirkt, während die als gravierendsten eingeschätzte Folge eines Bevölkerungsrückgangs, "Deutschlands kultureller Niedergang“, im Alltag kaum greifbar sein wird, sofern er nicht auf der Annahme fehlender Integration von Migranten beruht. Dies wäre allerdings keine Folge des Geburtenrückgangs. Nimmt man jedoch statt der individuellen Perspektive die des Staates ein, hat der Bevölkerungsrückgang erhebliche negative Auswirkungen, da er zu einem Rückgang von internationalem Einfluss und Macht beiträgt.

Ein wichtiges Ergebnis ist, dass bei der Analyse der Folgen eine analytische Trennung zwischen denen der Alterung und denen des Bevölkerungsrückgangs enorme Vorteile mit sich bringt, da beide sich hinsichtlich des Zeithorizonts, der Dynamik 
Folgen der dauerhaft niedrigen Fertilität in Deutschland •

79

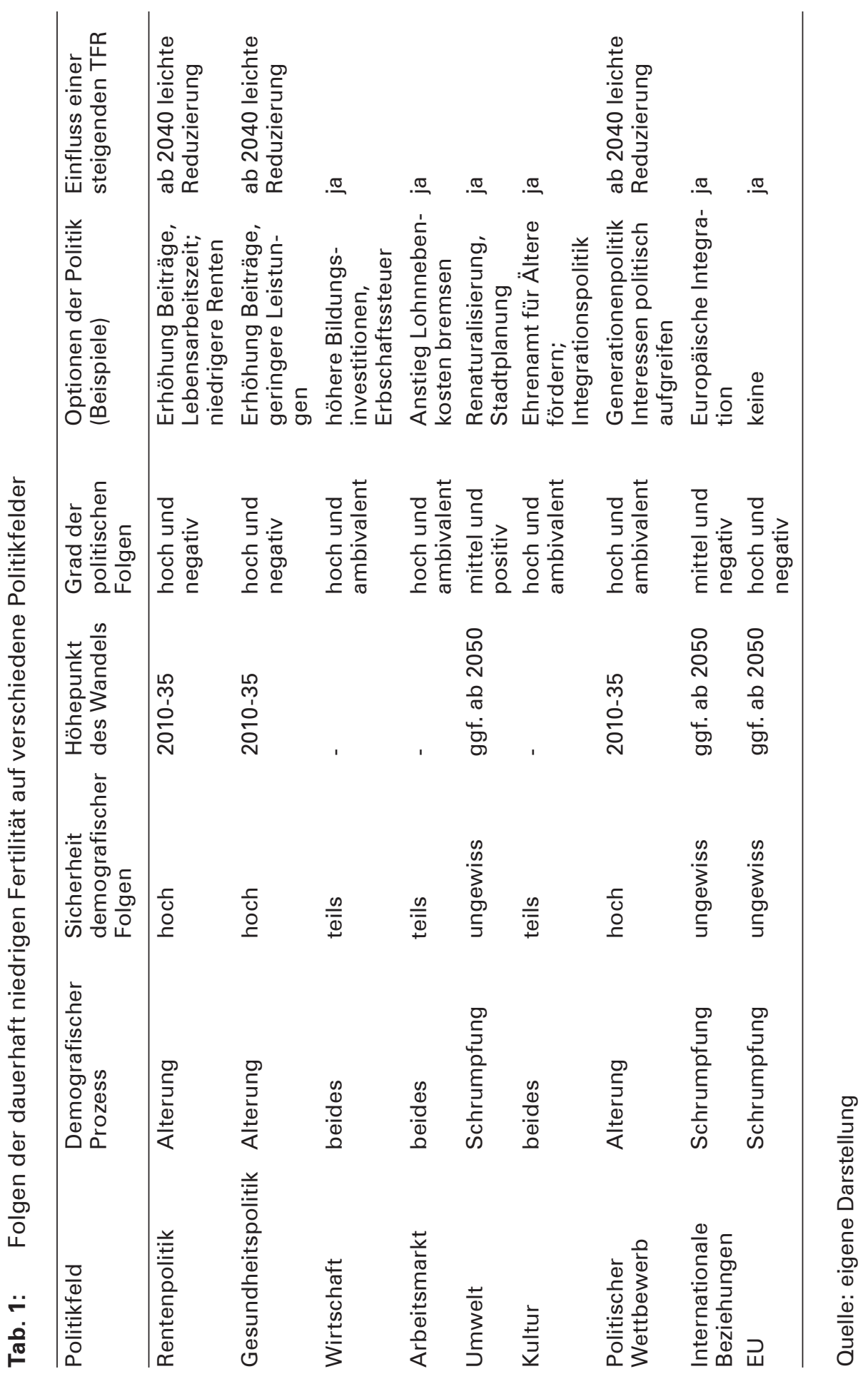


sowie des Einflusses von Migration und steigender Lebenserwartung grundsätzlich unterscheiden. Dies erklärt auch teilweise die heterogenen Interpretationsmuster, da in der Literatur häufig nicht exakt zwischen diesen demografischen Prozessen differenziert wird.

Bei Autoren, die negative Folgen des Zweiten Geburtenrückgangs verneinen (u.a. Müller 2004; Hondrich 2007), werden die oben gezeigten höchst negativen Konsequenzen für die sozialen Sicherungssysteme nicht als solche wahrgenommen. Angesichts der Verdoppelung des Altenquotienten wird mit Kompensationsmöglichkeiten durch zukünftiges Produktivitätswachstum und Migration argumentiert, jedoch ohne detaillierten Nachweis; beides wird in Relation zur Alterungsdynamik völlig überschätzt. Außerdem werden Entwicklungen der beiden Geburtenrückgänge vermengt und irreführende Analogien hergestellt. Auf der anderen Seite sind die Szenarien einiger Demografen, die die negativen Konsequenzen des Geburtenrückgangs betonen (u.a. Schmid et al. 2000; Birg 2003), deutlich überzeichnet. Dazu trägt die spezifische Auswahl von (negativen) Annahmen ebenso bei wie die fehlende Einordnung der Unsicherheit von langfristigen Modellrechnungen beim Bevölkerungsrückgang.

Wenn sich jedoch Bewertungen nicht nur auf den Geburtenrückgang, sondern auf den demografischen Wandel insgesamt beziehen, ist die normative neutrale Bewertung nicht unplausibel (u.a. Merke/ 2013). Denn dieser beinhalt mit der Zunahme der Lebenserwartung ein positives (Akademiegruppe Altern 2009; Kruse 2013) und mit dem Geburtenrückgang ein negatives Phänomen. Allerdings bedingen sich beide Phänomene nicht gegenseitig. Während von der zunehmenden Lebenserwartung alle Industrieländer betroffen sind, haben nur ein Teil der Länder wie Deutschland eine zu niedrige Geburtenrate.

Wie lassen sich Erkenntnisse der Fallstudie zu Deutschland auf andere Länder übertragen? Niedrige Fertilitätsraten implizieren nicht derartige Folgen, wenn sie nur für wenige Jahre so niedrig sind. In Deutschland dagegen hat sich der Rückgang der Geburtenzahl dynamisiert, da er sich bereits über zwei Generationen vollzieht. Vor dieser Entwicklung befinden sich gegenwärtig einige süd- und osteuropäische Staaten sowie Japan und Südkorea. Wenn das Low-Fertility-Syndrom in diesen Ländern ähnlich lange andauert wie in Deutschland, wird die Schrumpfung in Süd- und Osteuropa sogar noch stärker erfolgen, da Deutschland in den letzten Jahrzehnten von einer erheblichen Nettozuwanderung profitiert hat und diese Staaten teilweise von Abwanderungswellen betroffen sind. Da Deutschland ein Einwanderungsland ist, in das u.a. wegen der Aussiedlerwelle aus der (ehemaligen) Sowjetunion nach dem Fall des Eisernen Vorhangs ebenso wie infolge der Finanzkrise viele Menschen aus Süd- und Osteuropa gezogen sind, ist die durch das jahrzehntelange Geburtendefizit angelegte Schrumpfung kompensiert worden. In Japan ist dies nicht der Fall; zudem wird der Altenquotient höher steigen als in Deutschland, da dort die Lebenserwartung mehrere Jahre höher ist. Länder mit einem ausgeprägteren Babyboom wie Frankreich haben auch bei einer TFR um die Ausgleichsrate enorme Probleme mit dem Rentensystem, da der Altenquotient ebenfalls rapide ansteigt, wenn die Babyboomer-Generation das Rentenalter erreicht. 
Auf die Folgen für die Sozialsysteme wurde in Deutschland in den ersten zwei Jahrzehnten nach Beginn der Niedrigfertilität 1975 kaum oder nur in kleinteiligen Reformschritten reagiert. Die umfassendere Rentenreform, die das Renteneintrittsalter um zwei Jahre erhöht hat, wurde 2007 verabschiedet, also im 33. Jahr mit einer TFR unterhalb von 1,5. Eine offizielle, ressortübergreifende Demografiestrategie wurde 2012, im 38. Jahr als Low-Fertility-Land vorgelegt. Je stärker der Altersstruktureffekt und der Bevölkerungsrückgang sichtbar werden, desto intensiver wird die politische und mediale Debatte zur Demografie. Der deutsche Demografiediskurs wird, wie gezeigt wurde, mit erheblichen Übertreibungen und Extrempositionen geführt. Dies ist möglicherweise für andere Länder nicht so übertragbar, da die Erfahrungen mit pronatalistischen Missbrauch in Nazi-Deutschland einen langen Schatten auf den Diskurs geworfen haben. Andererseits ist die Debatte über demografische Folgen kompliziert, wenn Wissenschaftler bestimmte Entwicklungen benennen können, während die anderen Bürger noch keine Folgen sehen. Die Auswirkungen für Wirtschaft, Arbeitsmarkt, Umwelt und den politischen Wettbewerb lassen sich auch weitgehend auf andere Länder übertragen, sofern die niedrige Fertilität dort ähnlich lange anhält. Die Auswirkung auf die EU-Machtbalance ist bei Deutschland besonders gravierend, da Deutschland bezüglich der Größe von Wirtschaft und Bevölkerung dominiert und bei einem starken Bevölkerungsrückgang die politische Führungsposition verlieren könnte. Bei anderen Ländern wie Estland sind dagegen die kulturellen Folgen immens, da die Träger der Sprache und Erinnerungsgemeinschaft dort relativ klein sind.

Da es sich beim Auftreten von dauerhaft niedriger Fertilität um ein Phänomen neuer Dimension handelt, sind viele Folgen noch ungewiss und Gegenstand von zukünftiger Forschung. Einwanderung kann, möglicherweise auf Kosten zusätzlicher Probleme, den Bevölkerungsrückgang bremsen, nicht jedoch die Alterung. Die Analyse der Folgen zeigt, dass Länder wie Deutschland ein fundamentales Interesse an zukünftig höheren Geburtenraten haben. Das langfristige Wirkungspotenzial eines familienpolitischen Policymix auf die TFR (Bujard 2011; Luci-Greulich/Thévenon 2013) macht die Familienpolitik zu einem Schlüsselpolitikfeld der Zukunft. Allerdings lassen sich die Auswirkungen auf die Sozialversicherungen für die nächsten zwei Jahrzehnte nicht mehr demografisch abwenden. Steigt aber die Geburtenrate wieder auf Bestandserhaltungsniveau an, könnte der Altenquotient 2060 um $26 \%$ niedriger sein als bei konstanter TFR. Dies würde dauerhaft Spielräume für Rentensteigerungen für die nach 1970 geborenen Generationen implizieren. Zur Abwendung des möglichen Bevölkerungsrückgangs wäre ein Anstieg der TFR die zentrale Stellschraube. Auch wenn sie erst in ein, zwei oder drei Jahrzehnten anstiege, ließen sich die Negativszenarien einer exponentiell rückläufigen Bevölkerung abwenden.

\section{Danksagung}

Der Autor dankt Christian Fiedler, Frank Micheel, Harun Sulak und Stefan Wurster sowie zwei anonymen Gutachter(inne)n für wertvolle Hinweise. 


\section{Literatur}

Akademiegruppe Altern in Deutschland 2009: Gewonnene Jahre. Stuttgart: Wissenschaftliche Verlagsgesellschaft.

Barlösius, Eva; Schiek, Daniela (Hrsg.) 2007: Demographisierung des Gesellschaftlichen. Analysen und Debatten zur demographischen Zukunft Deutschlands. Wiesbaden: VS Verlag.

Basten, Stuart; Sobotka, Tomáš; Zeman, Krystof 2013: Future Fertility in Low Fertility Countries. Vienna Institute of Demography Working Papers 5/2013.

Bergmann, Michael; Konzelmann, Laura; Rattinger, Hans 2012: Deutschland auf dem Weg in die „Rentner Demokratie“? In: Politische Vierteljahresschrift 53: 371-393.

Birg, Herwig 2003: Die demographische Zeitenwende. 3. Auflage. München: Beck.

Blue, Laura; Espenshade, Thomas J. 2011: Population Momentum Across the Demographic Transition. In: Population and Development Review 37,4: 721-747 [doi: 10.1111/j.1728-4457.2011.00454.x].

Bongaarts, John 2001: Fertility and Reproductive Preferences in Post-Transitional Societies. In: Population and Development Review 27: 260-281.

Bujard, Martin 2011: Geburtenrückgang und Familienpolitik. Baden-Baden: Nomos.

Bujard, Martin et al. 2012: (Keine) Lust auf Kinder? - Geburtenentwicklung in Deutschland [http://www.bib-demografie.de/geburtenentwicklung2012, 05.05.2015].

BMI (Bundesministerium des Innern) 2012: Demografiebericht. Berlin: BMI.

BMI (Bundesministerium des Innern) 2013: Jedes Alter zählt. Demografiestrategie der Bundesregierung. Berlin: BMI.

Busemeyer, Marius; Goerres, Achim; Weschle, Simon 2009: Attitudes towards redistributive spending in an era of demographic ageing: the rival pressures from age and income in 14 OECD countries. In: Journal of European Social Policy 19,3: 195-212 [doi: 10.1177/0958928709104736].

Castles, Francis G. 2004: The Future of the Welfare State. Crisis Myths and Crisis Realities. Oxford: University Press.

CDU, CSU, SPD 2013: Deutschlands Zukunft gestalten. Koalitionsvertrag zwischen CDU, CSU und SPD [http://www.cdu.de/artikel/der-koalitionsvertrag-von-cdu-csu-und-spd, 16.12.2013].

Davis, Kingsley 1945: The World Demographic Transition. In: The Annals of The American Academy of Political and Social Science 237: 1-11 [doi: 10.1177/000271624523700102].

Doblhammer, Gabriele; Scholz, Rembrandt (Hrsg.) 2010: Ageing, Care Need and Quality of Life. Wiesbaden: VS Verlag.

Dudel, Christian 2014: Vorausberechnung von Verwandtschaft. Opladen: Barbara Budrich.

Easterlin, Richard A. 1974: Does economic growth improve the human lot? In: David, Paul A.; Reder, Melvin W. (Hrsg.): Nations and households in economic growth: Essays in honor of Moses Abramovitz. New York: Academic Press: 89-125.

Ette, Andreas; Ruckdeschel, Kerstin; Unger, Rainer (Hrsg.) 2010: Potenziale intergenerationaler Beziehungen. Chancen und Herausforderungen für die Gestaltung des demografischen Wandels. Beiträge zur Bevölkerungswissenschaft, Band 40. Würzburg: Ergon. 
Ewing, Brad et al. 2010: The Ecological Footprint Atlas 2010. Oakland: Global Footprint Network.

Friedman, Milton 1968: The Role of Monetary Policy. In: American Economic Review 58: 1-17.

Goerres, Achim 2007: Can We Reform the Welfare State in Times of "Grey" Majorities? In: MPIfG Working Paper 07/5.

Goldstein, Joshua; Rößger, Felix; Jaschinski, Ina; Prskawetz, Alexia 2011: Fertility Forecasting in the German-speaking World: Recent Experience and Opportunities for Improvement. In: Comparative Population Studies 36,2-3: 661-692 [doi: 10.4232/10. CPoS-2011-09en].

Höhn, Charlotte; Ette, Andreas; Ruckdeschel, Kerstin 2006: Kinderwünsche in Deutschland. Stuttgart: Robert Bosch Stiftung.

Hondrich, Karl 2007: Weniger sind mehr. Frankfurt: Campus.

Hüther, Michael; Naegele, Gerhard 2013: Demografiepolitik. Wiesbaden: Springer VS.

Huntington, Samue/ 1996: Kampf der Kulturen. München: Siedler.

Imhof, Arthur 1981: Die gewonnenen Jahre. München: Beck.

Kaufmann, Franz-Xaver 2005: Schrumpfende Gesellschaft. Frankfurt: Suhrkamp.

Keohane, Robert O.; Nye, Joseph S. 1977: Power and Interdependence. World Politics in Transition. Boston/Toronto: Little, Brown and Company.

Kielmansegg, Peter Graf 1995: Läßt sich die Europäische Union demokratisch verfassen? In: Weidenfeld, Werner (Hrsg.): Reform der Europäischen Union. Gütersloh: Bertelsmann.

Kolk, Martin; Cownden, Daniel; Enquist, Magnus 2014: Correlations in fertility across generations - can low fertility persist? Proceedings of the Royal Society B, 281: 20132561 [doi: 10.1098/rspb.2013.2561].

Kruse, Andreas 2013: Alternde Gesellschaft - eine Bedrohung? Berlin: Lambertus.

Kühntopf, Stephan; Stedtfeld, Susanne 2012: Wenige junge Frauen im ländlichen Raum. BiB Working Paper 3/2012. Wiesbaden: Bundesinstitut für Bevölkerungsforschung.

Lee, Ronald; Mason, Andrew 2007: Population Aging, Wealth, and Economic Growth: Demographic Dividends and Public Policy. WESS Background Paper.

Luci-Greulich, Angela; Thévenon, Olivier 2013: The Impact of Family Policies on Fertility Trends in Developed Countries. In: European Journal of Population 29,4: 387-416 [doi: 10.1007/s10680-013-9295-4].

Lutz, Wolfgang; Cuaresma, Jesus Crespo; Sanderson, Warren 2008: The demography of educational attainment and economic growth. In: Science 319,5866: 1047-1048 [doi: 10.1126/science.1151753].

Mayer, Tilman 2012: Demografiepolitik. BDF Working Paper 2-2012. Berlin: BDF.

Meadows, Donella H. et al. 1972: The Limits to Growth. New York: Universe Books.

Merkel, Angela 2012: Rede anlässlich des "Demografiegipfels“. 4.10.2012 [http://www. bundesregierung.de/Content/DE/Rede/2012/10/2012-10-04-bkin-demografiegipfel. html, 6.11.2013].

Merkel, Angela 2013: Rede beim zweiten Demografiegipfel. 14.5.2013 [http://www. bundesregierung.de/Content/DE/Rede/2013/05/2013-05-14-merkel-demografie.html, 6.11.2013]. 
Micheel, Frank 2013: Löst ein höheres Geburtenniveau die Problematik des abnehmenden Pflegepotenzials? In: Bundesgesundheitsblatt 56: 1104-1111 [doi: 10.1007/s00103013-1750-1]

Morgenthau, Hans 1963 [e. A. 1948]: Politics Among Nations. 3. Ed. New York: Knopf.

Müller, Albrecht 2004: Die Reformlüge. 40 Denkfehler, Mythen und Legenden, mit denen Politik und Wirtschaft Deutschland ruinieren. München: Droemer.

Münch, Ursula; Wilkoszewski, Harald 2006: Demografischer Wandel und Machtverteilung in Europa. In: Jahrbuch des Föderalismus. Hrsg. EZFF. Baden-Baden: Nomos: 533-551.

OECD 1998: The Macroeconomic Implications of Ageing in a Global Context. Economic Department Working Papers No. 193. Paris: OECD.

OECD 2014: OECD Family Database [www.oecd.org/social/family/database, 24.1.2014].

Rürup, Bert; Schmidt, Renate 2003: Nachhaltige Familienpolitik im Interesse einer aktiven Bevölkerungspolitik. Berlin: BMFSFJ.

Rürup, Bert; Ranscht, Anja 2009: Familienpolitik und soziale Sicherung. In: Biedenkopf, Kurt; Bertram, Hans; Niejahr, Elisabeth (Hrsg.): Starke Familie. Stuttgart: Robert Bosch Stiftung: 106-119.

Sánchez Gassen, Nora E. 2015: Germany's future electors. Wiesbaden: Springer VS.

Sander, Nikola 2014: Internal Migration in Germany, 1995-2010: New Insights into EastWest Migration and Re-urbanisation. In: Comparative Population Studies 39,2: 217 246 [doi: 10.12765/CPoS-2014-04en].

Scharpf, Fritz 2001: The Viability of Advanced Welfare States in the International Economy. In: Leibfried, Stephan (Hrsg.): Welfare State Futures. Cambridge: University Press: $123-150$

Scherbov, Sergei; Mamolo, Marija; Lutz, Wolfgang 2008: Probabilistic Population Projections for the 27 EU Member States. European Demographic Research Papers 2. Vienna: VID.

Schmid, Josef; Heigl, Andreas; Mai, Ralf 2000: Sozialprognose. Die Belastung der nachwachsenden Generation. München: Olzog.

Schmidt, Manfred G. 2005: Sozialpolitik in Deutschland. 3. Auflage, Opladen: Leske + Budrich.

Schirrmacher, Frank 2004: Das Methusalem-Komplott, München: Blessing.

Schultz, Theodore W. 1971: Investment in Human Capital. New York: The Free Press.

Sen, Amartya K. 1993: Capability and Well-Being. In: Sen, Amartya K.; Nussbaum, Martha (Hrsg.): The Quality of Life. Oxford: University Press: 30-53.

Sinn, Hans-Werner 2003: Das demografische Defizit. In: Leipert, Christian (Hrsg.): Demographie und Wohlstand. Opladen: Leske + Budrich: 57-88.

Sinn, Hans-Werner 2013: Das demographische Defizit - die Fakten, die Folgen, die Ursachen und die Politikimplikationen. In: ifo Schnelldienst 66,21: 1-23.

Smith, Adam 1776: An Inquiry into the Nature and Causes of the Wealth of Nations. Dublin: Printed for Messrs.

Sobotka, Tomás 2008: Overview Chapter 7: The rising importance of migrants for childbearing in Europe. In: Demographic Research 19, 9: 225-248 [doi: 10.4054/ DemRes.2008.19.9].

Solow, Robert M. 1956: A Contribution to the Theory of Economic Growth. In: The Quarterly Journal of Economics 70: 65-94. 
Statistisches Bundesamt 2009a: Bevölkerung Deutschlands bis 2060. 12. koordinierte Bevölkerungsvorausberechnung. Wiesbaden: Destatis.

Statistisches Bundesamt 2009b: Bevölkerung Deutschlands bis 2060. Ergebnisse der 12. koordinierten Bevölkerungsvorausberechnung. Excel Datei. Wiesbaden: Destatis.

Statistisches Bundesamt 2013a: Geburtentrends und Familiensituation in Deutschland. Wiesbaden: Destatis.

Statistisches Bundesamt 2013b: Bevölkerung. Wanderungen Deutschland [https:// www.destatis.de/DE/ZahlenFakten/Indikatoren/LangeReihen/Bevoelkerung/Irbev07. html, 07.01.2014].

Steinführer, Annett; Küpper, Patrick; Tautz, Alexandra 2014: Adapt and Cope: Strategies for Safeguarding the Quality of Life in a Shrinking Ageing Region. In: Comparative Population Studies 39,2: 345-370 [doi: 10.12765/CPoS-2014-07en].

Stock, Günter et al. (Hrsg.) 2012: Zukunft mit Kindern. Frankfurt: Campus.

Striessnig, Erich; Lutz, Wolfgang 2013: Can below-replacement fertility be desirable? In: Empirica 40,3: 409-425 [doi: 10.1007/s10663-013-9213-3].

Swiaczny, Frank 2014: How Much Influence Can We Have on Demographic Trends? In: Journal of Social Demogracy, International Quarterly Edition 2014, 1: 27-30.

UN (United Nations) 2000: Replacement Migration: Is it a Solution to Declining and Ageing Populations? New York: UN.

UN (United Nations) 2012: World Population Prospects: The 2012 Revision. New York: UN.

van de Kaa, Dirk 1987: Europe's Second Demographic Transition. In: Population Bulletin 42. Washington DC: Population Reference Bureau.

Walter, Norbert et al. 2013: Die Zukunft der Arbeitswelt. Stuttgart: Robert Bosch Stiftung.

Weidenfeld, Werner 1995: Europa - Weltmacht im Werden. In: Internationale Politik 5-1995: 17-22.

Wöhlcke, Manfred; Höhn, Charlotte; Schmid, Susanne 2004: Demografische Entwicklungen in und um Europa. Politische Konsequenzen. Baden-Baden: Nomos.

World Bank 2014: World Development Indicators [http://data.worldbank.org/data-catalog/world-development-indicators, 1.8.2014].

Eine Übersetzung dieses begutachteten und vom Autor autorisierten deutschen Originaltextes durch das Bundesinstitut für Bevölkerungsforschung ist unter dem Titel "Consequences of Enduring Low Fertility - A German Case Study. Demographic Projections and Implications for Different Policy Fields", DOI 10.12765/CPOS-2015-06en bzw. URN urn:nbn:de:bib-cpos-2015-06en2, auf http://www.comparativepopulationstudies.de verfügbar.

Eingegangen am: 01.08.2014

Angenommen am: 24.02.2015

Dr. Martin Bujard ( $\varangle$ ). Bundesinstitut für Bevölkerungsforschung. Wiesbaden, Deutschland.E-Mail: Martin.Bujard@bib.bund.de

URL: http://www.bib-demografie.de/bujard 


\section{Comparative Population Studies}

WWW.comparativepopulationstudies.de

ISSN: 1869-8980 (Print) - 1869-8999 (Internet)

Published by / Herausgegeben von

Prof. Dr. Norbert F. Schneider

Federal Institute for Population Research

D-65180 Wiesbaden / Germany

\section{Managing Editor /}

Verantwortlicher Redakteur

Frank Swiaczny

\section{Assistant Managing Editor /}

Stellvertretende Redakteurin

Katrin Schiefer

\section{Copy Editor (German) /}

Lektorat (deutsch)

Dr. Evelyn Grünheid

\section{Layout / Satz}

Beatriz Feiler-Fuchs

E-mail:cpos@bib.bund.de

\section{Scientific Advisory Board /}

Wissenschaftlicher Beirat

Paul Gans (Mannheim)

Johannes Huinink (Bremen)

Michaela Kreyenfeld (Rostock)

Marc Luy (Wien)

Clara H. Mulder (Groningen)

Notburga Ott (Bochum)

Peter Preisendörfer (Mainz)

Zsolt Spéder (Budapest)
Board of Reviewers / Gutachterbeirat Martin Abraham (Erlangen)

Laura Bernardi (Lausanne)

Hansjörg Bucher (Bonn)

Claudia Diehl (Konstanz)

Andreas Diekmann (Zürich)

Gabriele Doblhammer-Reiter (Rostock) Jürgen Dorbritz (Wiesbaden)

Anette Eva Fasang (Berlin)

E.-Jürgen Flöthmann (Bielefeld)

Alexia Fürnkranz-Prskawetz (Wien)

Beat Fux (Salzburg)

Joshua Goldstein (Berkeley)

Karsten Hank (Köln)

Sonja Haug (Regensburg)

Hill Kulu (Liverpool)

Aart C. Liefbroer (Den Haag)

Kurt Lüscher (Konstanz)

Emma Lundholm (Umeå)

Nadja Milewski (Rostock)

Dimiter Philipov (Wien)

Roland Rau (Rostock)

Tomáš Sobotka (Wien)

Jeroen Spijker (Barcelona)

Olivier Thévenon (Paris)

Helga de Valk (Brussel)

Heike Trappe (Rostock)

Michael Wagner (Köln) 